\title{
A New Crack Tip Element for the Phantom-Node Method with Arbitrary Cohesive Cracks
}

\begin{tabular}{|c|c|}
\hline Journal: & International Journal for Numerical Methods in Engineering \\
\hline Manuscript ID: & NME-May-07-0252.R1 \\
\hline Wiley - Manuscript type: & Research Article \\
\hline $\begin{array}{r}\text { Date Submitted by the } \\
\text { Author: }\end{array}$ & $31-$ Oct-2007 \\
\hline Complete List of Authors: & $\begin{array}{l}\text { Rabczuk, Timon; University of Canterbury, Department of } \\
\text { Mechanical Engineering } \\
\text { Zi, Goangseup; Korea University, Department of Civil, } \\
\text { Environmental and Architectural Engineering } \\
\text { Gerstenberger, Axel; Technical University of Munich, Institute for } \\
\text { Numerical Mechanics } \\
\text { Wall, Wolfgang; Technical University of Munich, Chair for } \\
\text { Computational Mechanics; Technical University of Munich, Chair for } \\
\text { Computational Mechanics }\end{array}$ \\
\hline Keywords: & $\begin{array}{l}\text { Cracks, Hansbo and Hansbo's approach, Phantom node method, } \\
\text { Dynamic fracture }\end{array}$ \\
\hline
\end{tabular}

\section{(5) ScholarONE" \\ Manuscript Central}




\title{
A New Crack Tip Element for the Phantom-Node Method with Arbitrary Cohesive Cracks
}

\author{
Timon Rabczuk ${ }^{1}$, Goangseup $\mathrm{Zi}^{2, *}$, Axel Gerstenberger ${ }^{3}$, and Wolfgang A. Wall ${ }^{3}$ \\ 1 Department of Mechanical Engineering, University of Canterbury, Christchurch, New Zealand \\ 2 Department of Civil, Environmental \&5 Architectural Engineering, Korea University, 5 Ga 1, An-Am Dong, \\ Sung-Buk Gu, Seoul, 136-701, Korea \\ 3 Institute for Numerical Mechanics, Technical University of Munich, Boltzmannstr. 15, D-85748 Garching \\ b. Munich, Germany
}

\section{SUMMARY}

We have developed a new crack tip element for the phantom node method. In this method, a crack tip can be placed inside an element. Therefore cracks can propagate almost independent of the finite element mesh. We developed two different formulations for the three-node triangular element and four-node quadrilateral element, respectively. Although this method is well suited for the one-point quadrature scheme, it can be used with other general quadrature schemes. We provide some numerical examples for some static and dynamic problems. Copyright (c) 2000 John Wiley \& Sons, Ltd.

KEY WORDs: Cracks; Hansbo and Hansbo's approach; Phantom node method; Dynamic fracture

\section{INTRODUCTION}

The extended finite element method (XFEM) $[11,35]$ is a powerful tool for analyzing problems that involve strong and weak discontinuities such as cracks [15-17, 54], fluid-structure interactions [28] or material interfaces e.g. in two-phase flow [19]. For crack problems, many features are incorporated into the XFEM formulation such as cohesive cracks [1,33], branching and joining cracks $[16,53]$, cohesive cracks whose tip end in an element [52], and explicit time integration for XFEM including an efficient and simple mass lumping scheme (based on spectral analysis) $[29,30]$ just to name a few of them.

Recently, an alternative method to the XFEM has been proposed by Hansbo and Hansbo [24]. The basic difference from the original XFEM is the way that the approximation space is enriched. In the original XFEM, additional degrees of freedom are introduced into the

* Correspondence to: Department of Civil, Environmental \& Architectural Engineering, Korea University, 5 Ga 1, An-Am Dong, Sung-Buk Gu, Seoul, 136-701, Korea; Tel.: +82-2-3290-3324; g-zi@korea.ac.kr

Contract/grant sponsor: The Agency for Defense Development (ADD), and the Korea Institute of Construction \& Transportation Technology Evaluation and Planning (KICTTEP); contract/grant number: ADD-06-05-06 and 05-CTRM-D04-03, respectively 


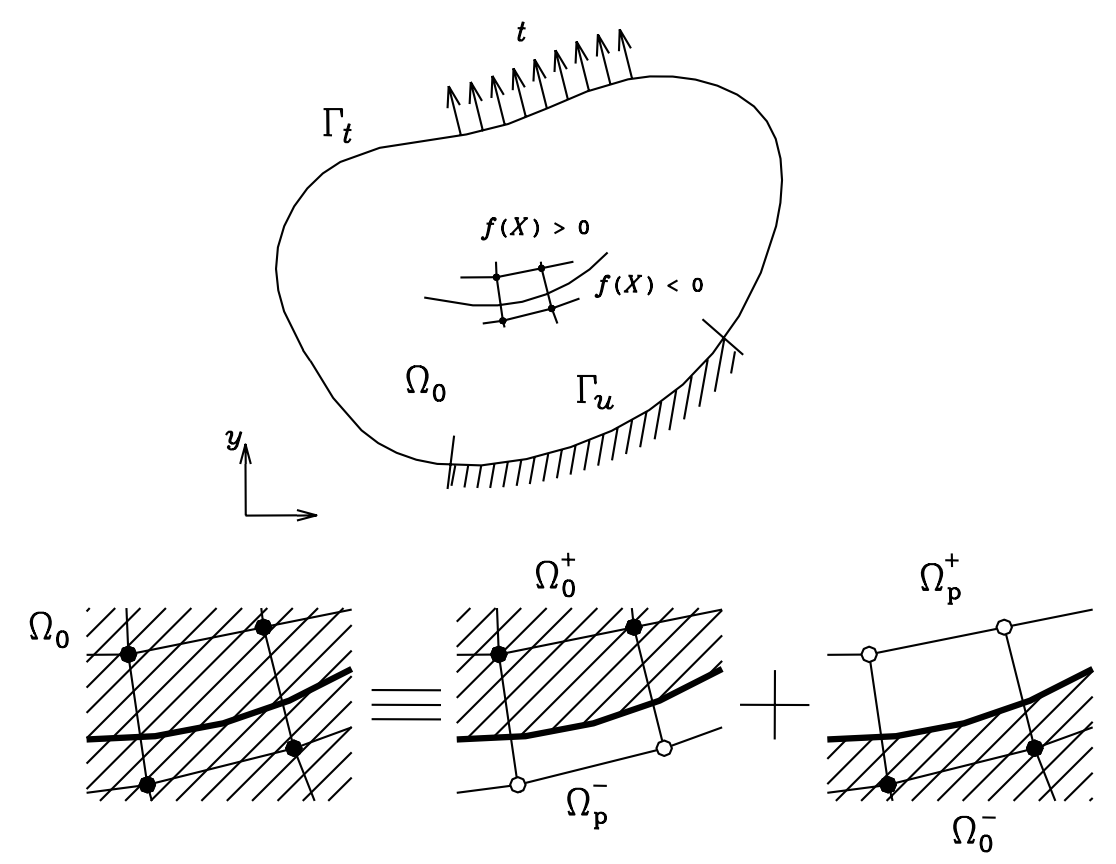

Figure 1. The principle of the phantom node method in which the hatched area is integrated to build the discrete momentum equation; the solid circles represent real nodes and the empty ones phantom nodes.

variational formulation. These additional degrees of freedom solely determine the crack kinematics, i.e. the jump in the displacement field. Hansbo and Hansbo [24] suggest a method where the crack kinematics is obtained by overlapping elements instead of introducing additional degrees of freedom. Though it has been shown by Song et al. [44] that the method of Hansbo and Hansbo [24] is equivalent to the original XFEM, the formulation by Hansbo and Hansbo [24] has particular advantages with respect to the implementation of the method. First, since all degrees of freedom are physical, the mass matrix can be obtained by a standard row sum procedure that is especially useful in explicit dynamic codes. Second, the idea of Hansbo and Hansbo [24] is easier to implement into commercial finite element codes since no additional degrees of freedom are introduced that increase in number when the crack grows [45]. While Hansbo and Hansbo [24] has given a rather a theoretic framework, Mergheim et al. [31] have implemented the idea of Hansbo and Hansbo [24] in a static setting in two dimensions and also in three dimensions [32]. Song et al. [45] has introduced a two-dimensional phantom-node method in two dimensions. This concept has also been used in a shell-framework in Areias et al. [2].

We follow the phantom-node method in Song et al. [45] and extend their approach to model crack tips within an element. In Song et al. [45], the crack has to cross an entire element. We propose this idea for triangular and quadrilateral elements using reduced integrated finite elements with hourglass control [10]. Thanks to the absence of an enrichment, it is simple to incorporate concepts such as the enhanced assumed strain (EAS) into the new formulation. 

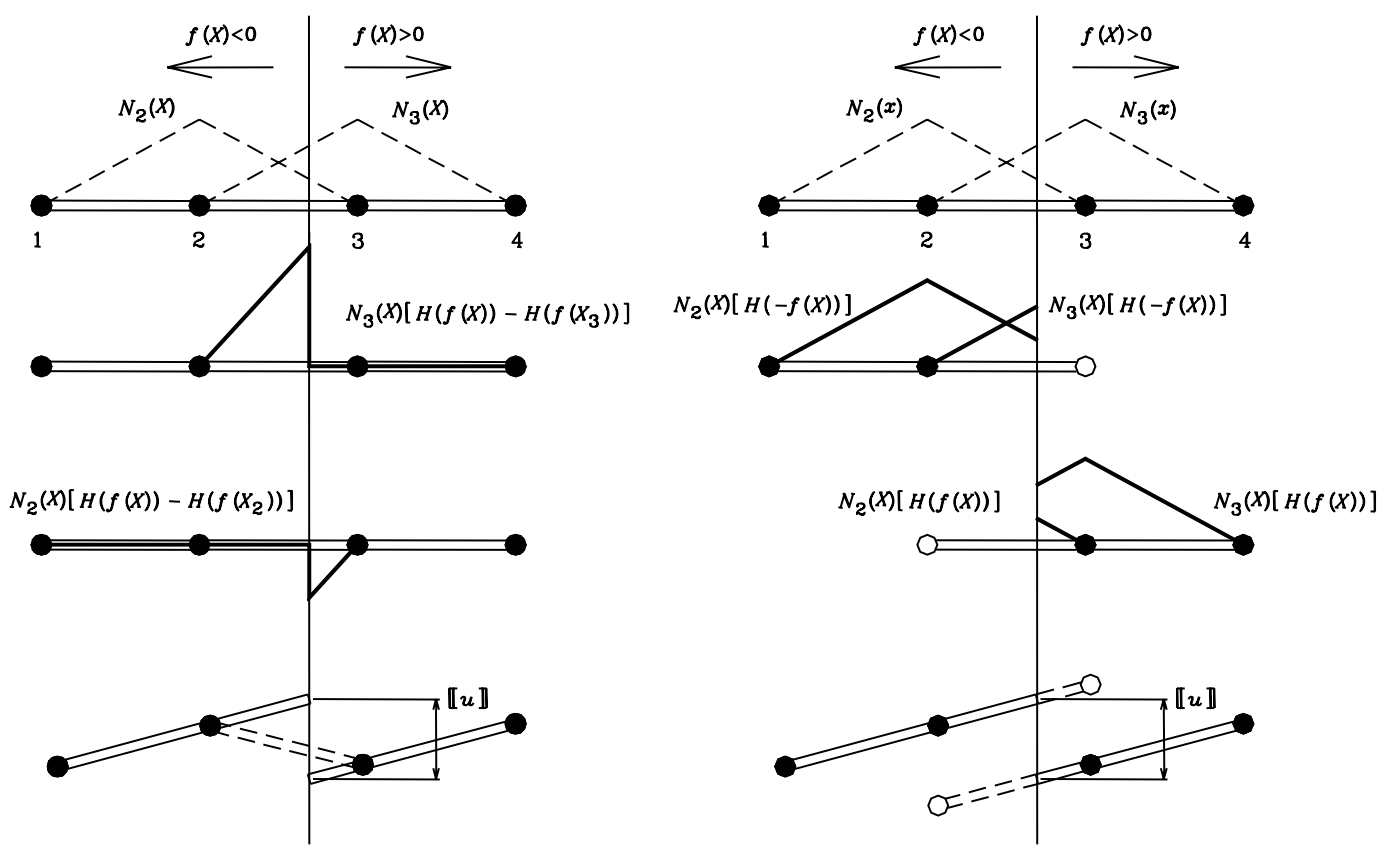

Figure 2. The standard XFEM vs. phantom node method.

\section{A REVIEW OF THE PHANTOM NODE METHOD}

Consider a body that is cracked as shown in Fig. 1 and the corresponding finite element discretization. Because of the crack, there are cracked elements cut by the crack. To have a set of full interpolation bases, the part of the cracked elements which belongs in the real domain $\Omega_{0}$ are extended to the phantom domain $\Omega_{\mathrm{p}}$. Then the displacement in the real domain $\Omega_{0}$ can be interpolated by using the degrees of freedom for the nodes in the phantom domain $\Omega_{\mathrm{p}}$. The nodes are called the phantom nodes and marked by empty circles in Fig. 1. The approximation of the displacement field is then given by [45]:

$$
\mathbf{u}^{h}(\mathbf{X}, t)=\sum_{I \in\left\{\mathcal{W}_{0}^{+}, \mathcal{W}_{\mathrm{P}}^{-}\right\}} \mathbf{u}_{I}(t) N_{I}(\mathbf{X}) H(f(\mathbf{X}))+\sum_{J \in\left\{\mathcal{W}_{0}^{-}, \mathcal{W}_{\mathrm{P}}^{+}\right\}} \mathbf{u}_{J}(t) N_{J}(\mathbf{X}) H(-f(\mathbf{X}))
$$

where $f(\mathbf{X})$ is the signed distance measured from the crack, $\mathcal{W}_{0}^{+}, \mathcal{W}_{0}^{-}, \mathcal{W}_{\mathrm{P}}^{+}$and $\mathcal{W}_{\mathrm{P}}^{-}$are nodes belonging to $\Omega_{0}^{+}, \Omega_{0}^{-}, \Omega_{\mathrm{P}}^{+}$and $\Omega_{\mathrm{P}}^{-}$, respectively. $H(x)$ is the Heaviside function. As can be seen from Fig. 1, cracked elements have both real nodes and phantom nodes. The jump in the displacement field is realized by simply integrating only over the area from the side of the real nodes up to the crack, i.e. $\Omega_{0}^{+}$and $\Omega_{0}^{-}$. The shape functions for a one-dimensional overlapped phantom-node element are shown in Fig. 2 and compared to the original XFEM method. We note that there are certain similarities to the visibility method used originally in meshfree methods, [12-14, 36]. 


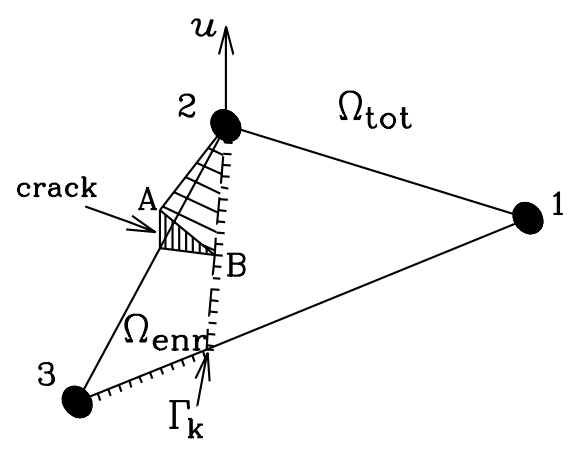

Figure 3. Zi and Belytschko [52]'s enriched displacement field.

\section{THE NEW TIP ELEMENT}

\subsection{Three-node triangular element}

In the cohesive crack model, the crack opening displacement $\llbracket \boldsymbol{u} \rrbracket$ should vanish at the crack tip without the stress singularity found in linear elastic fracture mechanics (LEFM). In the classical extended finite element method, the condition was fulfilled by using a nonsingular branch enrichment [34]. Recently, Zi and Belytschko [52] showed that the condition could be fulfilled with only the step enrichment for triangular elements. They used a different shape function for the enrichment field from the continuous displacement approximation so that the crack opening displacement vanished at the edge $\Gamma_{k}$ of the enriched shape function; see Fig. 3.

A simple crack tip element for the phantom node method can be devised easily in the extension line of Zi and Belytschko [52]'s method. First, expand the small triangle representing the enriched displacement in Fig. 4 to obtain a new triangle. The new triangular element $1 * 23^{*}$ is shown in Fig. 4a. Node 2 is shared by the two elements. The difference in the displacement of the two elements vanishes at $\Gamma_{k}$.

Integrating area $A_{1}$ in element 123 and $A_{2}$ in element $1^{*} 23^{*}$, we then have the same displacement field as in Fig. 3. It is trivial that the sum of $A_{1}$ and $A_{2}$ is equal to the area of element 123 . Note that there are only three physical nodes which are the solid circles in Fig. 4a. The nodal values of the extra degrees of freedom, marked by the empty circles in Fig. 4a should be determined from a kinematical constraint. For the case illustrated in Fig. 4a,b,c, displacement $\boldsymbol{u}^{*}$ is given by

$$
\begin{aligned}
& \xi_{1 \mathrm{P}}\left(\boldsymbol{u}_{1}^{*}-\boldsymbol{u}_{1}\right)+\left(1-\xi_{1 \mathrm{P}}\right)\left(\boldsymbol{u}_{3}^{*}-\boldsymbol{u}_{3}\right)=0 \\
& \left(\boldsymbol{u}_{2}^{*}-\boldsymbol{u}_{2}\right)=0
\end{aligned}
$$

in which $\left(\xi_{1 \mathrm{P}}, 0\right)$ is the intersection point of $\Gamma_{k}$ and edge 31 in the parent coordinate of the element; see Fig. 4b,c.

There can be another case as in Fig. 4 d such that node 3 is shared. The displacement $\boldsymbol{u}^{*}$ is

Int. J. Numer. Meth. Engng 2000; 00:1-6 


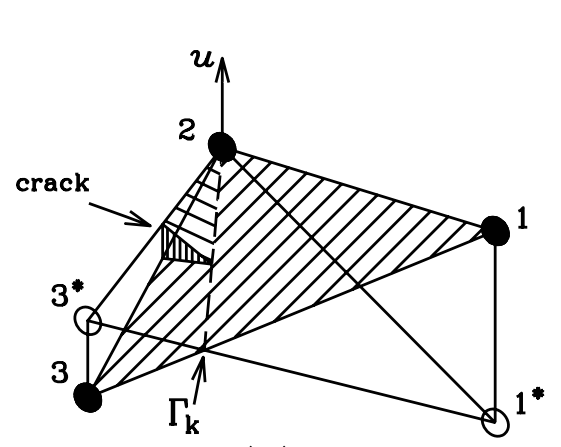

(a)

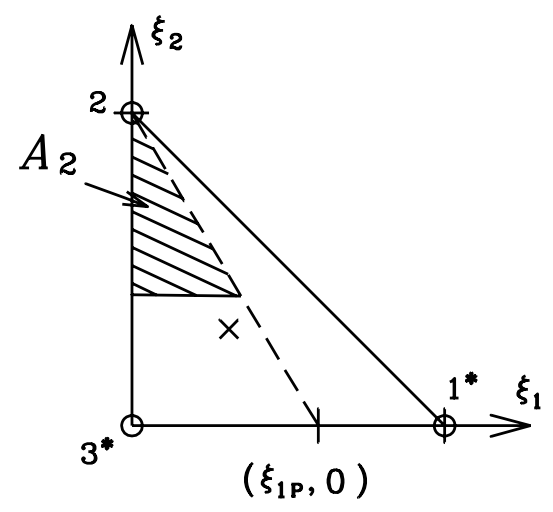

(c)

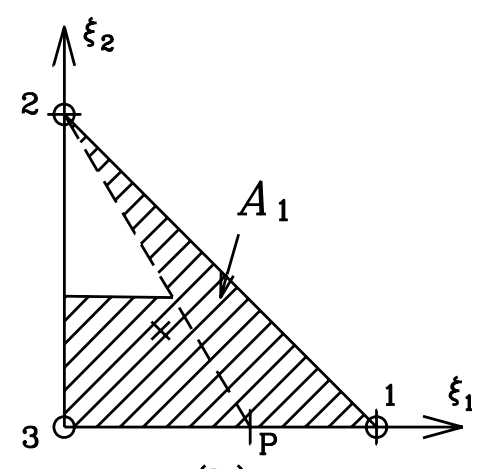

(b)

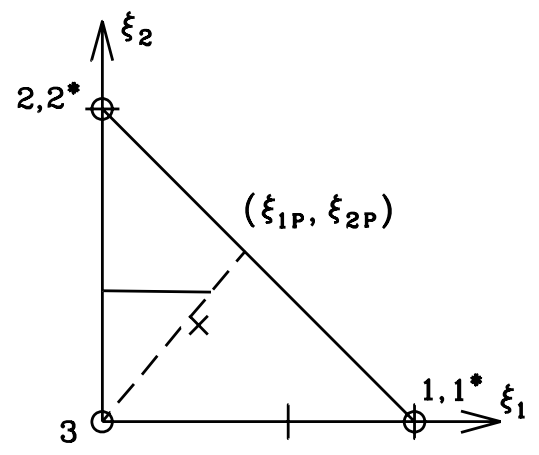

(d)

Figure 4. (a) A three-node tip element for the phantom node method in which solid circles represent physical nodes and empty symbols the extra degrees of freedom to be determined by a kinematical constraint, (b) and (c) the areas to be integrated and (d) another case of crack development.

given by

$$
\begin{aligned}
& \xi_{1 \mathrm{P}}\left(\boldsymbol{u}_{1}^{*}-\boldsymbol{u}_{1}\right)+\xi_{2 \mathrm{P}}\left(\boldsymbol{u}_{2}^{*}-\boldsymbol{u}_{2}\right)=0 \\
& \left(\boldsymbol{u}_{3}^{*}-\boldsymbol{u}_{3}\right)=0
\end{aligned}
$$

in which $\left(\xi_{1 \mathrm{P}}, \xi_{2 \mathrm{P}}\right)$ is the intersection point of $\Gamma_{k}$ and edge 12 in the parent coordinate of the element; see Fig. 4d. When the position of the crack tip is close to edge 12, this would produce a well-suited $\Gamma_{k}$. If the crack is too close to an element side or node, there is no need to close the crack within an element and the crack is closed as in the conventional phantom node method.

The approach mentioned above is not the only way. There is an alternative where three elements may be used; see Fig. 5. Again, the difference in the displacement of those elements vanishes at $\Gamma_{k}$. The sum of $A_{1}, A_{2}$ and $A_{3}$ is equal to the area of the triangle. The value of 


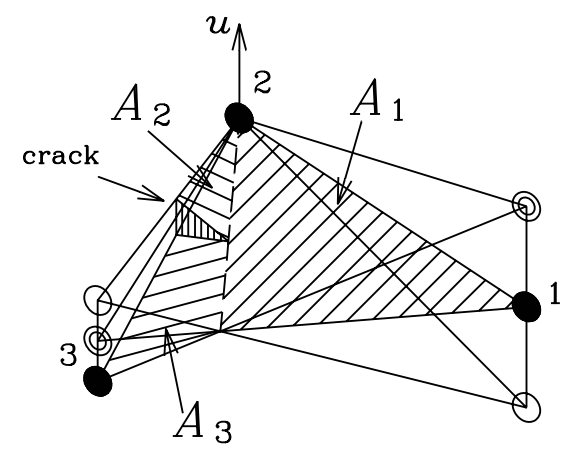

Figure 5. An alternative three-node tip element for the phantom node method in which solid circles represent physical nodes and empty symbols, the extra degrees of freedom to be determined by a kinematical constraint.

the extra degrees of freedom can be determined in a similar way as Eqs. (2) to (5).

To accurately integrate the hatched area in Fig. 4 or 5, it is inevitable to project internal variables stored in a set of quadrature points to another for general quadrature rules. The subtriangulation of a cracked element is commonly used for the numerical integration [34]. The standard quadrature scheme without the subtriangulation is also possible, in which there is no projection of internal variables [23, 48]. In that case, the use of a sufficiently high order quadrature is necessary for the reduction of the integration error due to the crack. If the one point quadrature is used, there is no need for the projection. The integration of the hatched area is carried out by, simply, the product of the area fraction $A_{1} / A$ or $A_{2} / A$ and the integration for the original triangle, in which $A$ is the area of the original triangle 123 .

The conventional phantom node method is used if the crack tip is too close to an edge or a node. A similar technique is employed in the standard XFEM techniques, Dolbow et al. [20].

\subsection{Four-node quadrilateral element}

The method developed with three-node triangular elements can be applied for four-node quadrilateral elements with a slight difference. The standard displacement field of a four-node quadrilateral element is bilinear. Therefore if we overlap two quadrilateral elements together, similar to the case with the three-node triangular elements, the intersection $\Gamma_{k}$ is not always a line. The only case in which $\Gamma_{k}$ is a line is when the intersection is parallel to one of the axes of the parent coordinates.

Let a crack always grow to the element from edge 41; see Fig. 6 . Consider four phantom nodes $3^{*}, 4^{*}, 1^{* *}$ and $2^{* *}$, and three rectangles $1234,123^{*} 4^{*}$ and $1^{* *} 2^{* *} 34$. The only case that there is a single line intersection $\Gamma_{k}$ is that $\Gamma_{k}$ is parallel to the axis of $\xi_{2}$. The intersection between $\Gamma_{k}$ and edge 34 or 12 is point $\left(\xi_{1 \mathrm{P}},-1\right)$ or $\left(\xi_{1 \mathrm{P}}, 1\right)$.

From the condition that the difference of the displacement must be equal to zero along $\Gamma_{k}$, we can devise a kinematical constraint corresponding to the configuration discussed above us 


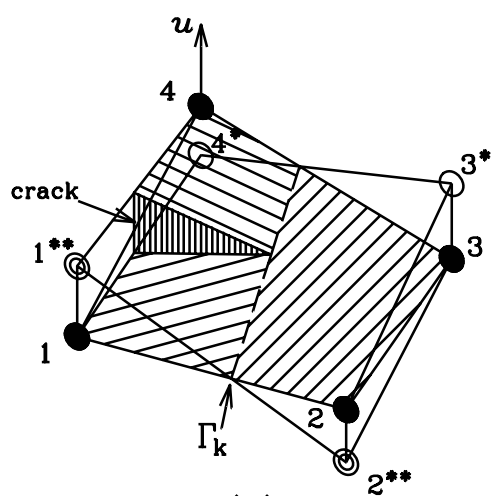

(a)

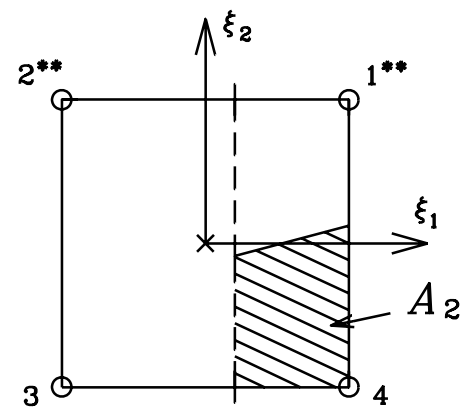

(c)

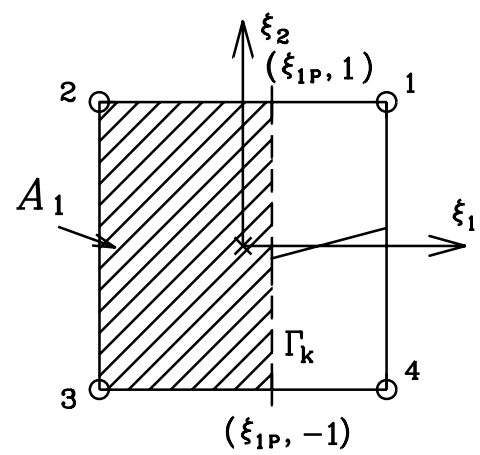

(b)

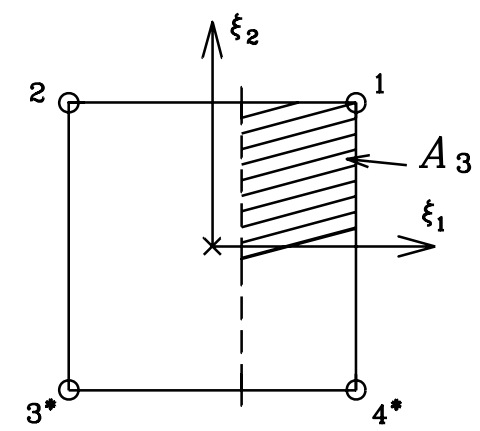

(d)

Figure 6. (a) A four-node tip element for the phantom node method in which solid circles represent physical nodes and empty symbols, the extra degrees of freedom to be determined by a kinematical constraint, and (b) and (c) the areas to be integrated.

given by

$$
\begin{aligned}
\boldsymbol{u}_{3}^{*} & =\boldsymbol{u}_{3}-k_{c}\left(\boldsymbol{u}_{4}^{*}-\boldsymbol{u}_{4}\right) \\
\boldsymbol{u}_{2}^{* *} & =\boldsymbol{u}_{2}-k_{c}\left(\boldsymbol{u}_{1}^{* *}-\boldsymbol{u}_{1}\right)
\end{aligned}
$$

in which $k_{c}=\left(1+\xi_{1 \mathrm{P}}\right) /\left(1-\xi_{1 P}\right)$. Note $\boldsymbol{u}_{4}^{*}$ and $\boldsymbol{u}_{1}^{* *}$ are determined from the continuity condition with the adjacent elements. If Belytschko and Bindemann [10]'s element is used as the base element, the one point quadrature can be adopted for the four-node quadrilateral element.

Int. J. Numer. Meth. Engng 2000; 00:1-6 


\section{DESCRIPTION OF CRACKS}

A crack can be described by piecewise linear line segments. The level set method is frequently used within the XFEM [see 46, 47, 52] and XEFG [40, 49, 50]. A level set function defines the surface of a discontinuity by a function of position $\boldsymbol{X}$ measured from the discontinuity. Note that this function does not necessarily have to be the signed distance function given by

$$
f_{J}(\boldsymbol{X})=\operatorname{sign}\left[\boldsymbol{n} \cdot\left(\boldsymbol{X}-\boldsymbol{X}_{J}\right)\right] \min \left\|\boldsymbol{X}-\boldsymbol{X}_{J}\right\| \text { with } \boldsymbol{X}_{J} \in \Gamma_{c}
$$

We do not explain the detailed crack tracing procedure with level sets in more detail but instead, refer the interested readers to the literature.

The jump in the displacement is given by

$$
\llbracket \boldsymbol{u}(\boldsymbol{X}) \rrbracket=\sum_{I \in\left\{\mathcal{W}_{0}^{+}, \mathcal{W}_{\mathrm{P}}^{-}\right\}} N_{I}(\boldsymbol{X}) \boldsymbol{u}_{I}-\sum_{J \in\left\{\mathcal{W}_{0}^{-}, \mathcal{W}_{\mathrm{P}}^{+}\right\}} N_{J}(\boldsymbol{X}) \boldsymbol{u}_{J}
$$

where $\mathcal{W}_{\mathrm{p}}$ is the set of nodes in phantom domain $\Omega_{\mathrm{p}}$. The normal part $\delta_{n}$, i.e. the crack opening and the tangential part $\llbracket \mathbf{u}(\boldsymbol{X}) \rrbracket_{\tau}$, the crack sliding is given by

$$
\begin{aligned}
\delta_{n} & =\boldsymbol{n} \cdot \llbracket \boldsymbol{u}(\boldsymbol{X}) \rrbracket \\
\delta_{t} & =\left\|\llbracket \boldsymbol{u}(\boldsymbol{X}) \rrbracket-\boldsymbol{n} \delta_{n}\right\|
\end{aligned}
$$

More details are given in Belytschko et al. [15]. If not mentioned otherwise, we only consider normal forces and neglect any mode II effect.

We use the Rankine criterion in order to initiate the crack, i.e. a crack is introduced once the tensile strength is exceeded.

\section{GOVERNING EQUATIONS}

\subsection{The momentum equations and the boundary conditions}

The strong form of the momentum equation in the total Lagrangian description is given by

$$
\varrho_{0} \ddot{\boldsymbol{u}}=\nabla_{0} \cdot \boldsymbol{P}+\varrho_{0} \boldsymbol{b} \quad \text { in } \quad \Omega_{0} \backslash \Gamma_{0}^{c}
$$

with boundary conditions:

$$
\begin{aligned}
\boldsymbol{u}(\boldsymbol{X}, t) & =\overline{\boldsymbol{u}}(\boldsymbol{X}, t) \text { on } \Gamma_{0}^{u} \\
\boldsymbol{n}^{0} \cdot \boldsymbol{P}(\boldsymbol{X}, t) & =\overline{\boldsymbol{t}}(\boldsymbol{X}, t) \text { on } \Gamma_{0}^{t} \\
\boldsymbol{n}_{0} \cdot \boldsymbol{P}^{-} & =\boldsymbol{n}_{0} \cdot \boldsymbol{P}^{+}=\boldsymbol{t}_{c 0} \text { on } \Gamma_{0}^{c} \\
\boldsymbol{t}_{c 0} & =\boldsymbol{t}_{c 0}(\llbracket \boldsymbol{u} \rrbracket) \text { on } \Gamma_{0}^{c}
\end{aligned}
$$

where $\varrho_{0}$ is the initial mass density, $\ddot{\boldsymbol{u}}$ is the acceleration, $\boldsymbol{P}$ denotes the nominal stress tensor, $\boldsymbol{b}$ designates the body force, $\overline{\boldsymbol{u}}$ and $\overline{\boldsymbol{t}}$ are the prescribed displacement and traction, respectively, $\boldsymbol{n}_{0}$ is the outward normal to the domain and $\Gamma_{0}^{u} \cup \Gamma_{0}^{t} \cup \Gamma_{0}^{c}=\Gamma_{0},\left(\Gamma_{0}^{u} \cap \Gamma_{0}^{t}\right) \cup\left(\Gamma_{0}^{t} \cap \Gamma_{0}^{c}\right) \cup\left(\Gamma_{0}^{c} \cap \Gamma_{0}^{u}\right)=$ $\emptyset$. Moreover, we assume that the stresses $\boldsymbol{P}$ at the crack surface $\Gamma_{0}^{c}$ are bounded. Since the stresses are not well defined in the crack, the crack surface $\Gamma_{0}^{c}$ is excluded from the domain $\Omega_{0}$ which is considered as an open set.

Int. J. Numer. Meth. Engng 2000; 00:1-6 


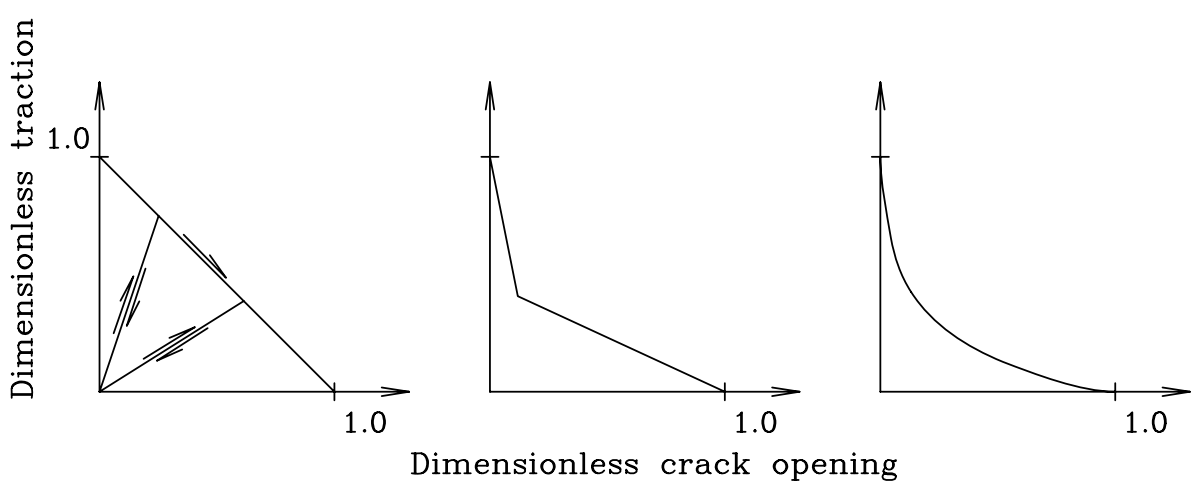

Figure 7. The types of the cohesive laws frequently used in practice; (a) linear (or triangular), (b) bilinear and (c) exponential cohesive laws.

\subsection{Constitutive equations}

We use Rankine type materials and the Johnson-Cook model [25]. The Johnson-Cook model [25] is based on $J_{2}$ plasticity but takes into account the strain rate and temperature effects. The effective yield stress of the Johnson-Cook model is given by

$$
\sigma_{Y}=\left(A+B \gamma^{n}\right)\left(1+C \ln \dot{\epsilon}^{*}\right)\left(1-T^{* m}\right)
$$

with $\dot{\epsilon}^{*}=\dot{\gamma} / \dot{\gamma}_{0}$ where $\gamma$ is the effective plastic strain and $\dot{\gamma}_{0}$ is the reference strain rate taken to be $1.0 / \mathrm{s}$ and

$$
T^{*}=\frac{T-T_{r}}{T_{m}-T_{r}}
$$

where $T_{r}$ is the reference temperature and $T_{m}$ is the melting temperature. We assume that the plastic deformation is completely transformed into heat, so $\beta=1$ for the temperature update:

$$
\Delta T=\int_{0}^{\gamma} \frac{\beta}{\varrho c_{v}} \sigma_{Y} d \gamma
$$

Batra and Gummalla [4] pointed out that the initial negative slope of the effective plastic stress-effective plastic strain curve highly determines the shear band initiation. They suggested the following form of the effective yield stress that gave better results in his computations:

$$
\sigma_{Y}=\max \left\{\left(A+B \gamma^{n}\right)\left(1+C \ln \dot{\epsilon}^{*}\right)\left[1-\delta \exp \left(\left(T-T_{0}\right) / \kappa_{0}-1\right)\right], 0\right\}
$$

in which $\delta$ is a parameter which controls the thermal softening in the model and $\delta=0.8$ is used. A crack is introduced or propagated when the stresses reaches a value of $2 A$ as assumed e.g. in Batra and coworkers' [5-8].

\subsection{Cohesive cracks}

In the cohesive crack model, there is no stress singularity near the crack tip and the traction on the crack surface is a function of the crack opening displacement. The cohesive crack models 
that are most popularly used in practice are shown in Fig. 7. For simplicity of the problem, the linear cohesive law is adopted in this study. Then the cohesive traction $\tau$ is given by

$$
\tau(\delta)=\left\{\begin{array}{lll}
\tau_{f}\left(1-\frac{\delta}{\delta_{c}}\right) & \text { if } & \delta \leq \delta_{c} \\
0 & \text { if } \quad \delta>\delta_{c}
\end{array}\right.
$$

in which $\delta$ is the crack opening displacement, $\delta_{c}$ is the critical crack opening displacement beyond which the cohesive traction is reduced to zero and $\tau_{f}$ is the strength of the material.

If a potential for the cohesive crack is defined, the unidirectional relation of Eq. (21) can be extended to general mixed mode problems, too [9, 18, 37, etc]. Here we consider only the mode I crack separation. The computational implementation of Eq. (21) including the unloading and reloading behaviors can be found in the literature [e.g. 16].

\subsection{The discrete governing eqthtengSCRETIZED EQUATIONS}

The weak form of the momentum equation is given by

$$
\delta W=\delta W_{\mathrm{int}}+\delta W_{\mathrm{kin}}-\delta W_{\mathrm{ext}}-\delta W_{\mathrm{coh}}
$$

in which $\delta W_{\mathrm{int}}, \delta W_{\mathrm{kin}}, \delta W_{\mathrm{ext}}, \delta W_{\mathrm{coh}}$ are the virtual works by the internal stress, the inertia force, the external traction and the cohesive traction, respectively. They are given by

$$
\begin{aligned}
\delta W_{\mathrm{int}} & =\int_{\Omega_{0} \backslash \Gamma_{0}^{c}}(\nabla \otimes \delta \boldsymbol{u})^{T}: \boldsymbol{P} \mathrm{d} \Omega_{0} \\
\delta W_{\mathrm{kin}} & =\int_{\Omega_{0} \backslash \Gamma_{0}^{c}} \varrho_{0} \delta \boldsymbol{u} \cdot \ddot{\boldsymbol{u}} \mathrm{d} \Omega_{0} \\
\delta W_{\mathrm{ext}} & =\int_{\Omega_{0} \backslash \Gamma_{0}^{c}} \varrho_{0} \delta \boldsymbol{u} \cdot \boldsymbol{b} \mathrm{d} \Omega_{0}+\int_{\Gamma_{0}^{t}} \delta \boldsymbol{u} \cdot \overline{\boldsymbol{t}}_{0} \mathrm{~d} \Gamma_{0} \\
\delta W_{\mathrm{coh}} & =\int_{\Gamma_{0}^{c}} \llbracket \delta \boldsymbol{u} \rrbracket \cdot \boldsymbol{\tau} \mathrm{d} \Gamma_{0}
\end{aligned}
$$


With the trial functions, Eq. (1) and the test functions, that have the same structure, and the crack opening displacement $\llbracket \boldsymbol{u} \rrbracket$ in Eq. (9), we obtain

$$
\begin{aligned}
& \delta W_{\text {int }}=\sum_{I \in\left\{\mathcal{W}_{0}^{+}, \mathcal{W}_{\mathrm{P}}^{-}\right\}} \delta \boldsymbol{u}_{I}^{T} \int_{\Omega_{0}^{+} \backslash \Gamma_{0}^{c}} \nabla_{0} N_{I}(\boldsymbol{X})^{T}: \boldsymbol{P} \mathrm{d} \Omega \\
& +\sum_{J \in\left\{\mathcal{W}_{0}^{-}, \mathcal{W}_{\mathrm{P}}^{+}\right\}} \delta \boldsymbol{u}_{J}^{T} \int_{\Omega_{0}^{-} \backslash \Gamma_{0}^{c}} \nabla_{0} N_{J}(\boldsymbol{X})^{T}: \boldsymbol{P} \mathrm{d} \Omega \\
& \delta W_{\text {kin }}=\sum_{I \in\left\{\mathcal{W}_{0}^{+}, \mathcal{W}_{\mathrm{P}}^{-}\right\}} \delta \boldsymbol{u}_{I}^{T} \sum_{K \in\left\{\mathcal{W}_{0}^{+}, \mathcal{W}_{\mathrm{P}}^{-}\right\}} \int_{\Omega_{0}^{+} \backslash \Gamma_{0}^{c}} \varrho_{0} N_{I}(\boldsymbol{X})^{T} \cdot N_{K}(\mathbf{X}) \mathrm{d} \Omega \ddot{\boldsymbol{u}}_{K} \\
& +\sum_{I \in\left\{\mathcal{W}_{0}^{-}, \mathcal{W}_{\mathrm{P}}^{+}\right\}} \delta \boldsymbol{u}_{I}^{T} \sum_{L \in\left\{\mathcal{W}_{0}^{-}, \mathcal{W}_{\mathrm{P}}^{+}\right\}} \int_{\Omega_{0}^{-} \backslash \Gamma_{0}^{c}} \varrho_{0} N_{I}(\mathbf{X})^{T} \cdot N_{L}(\boldsymbol{X}) \mathrm{d} \Omega \ddot{\boldsymbol{u}}_{L} \\
& +\sum_{J \in\left\{\mathcal{W}_{0}^{+}, \mathcal{W}_{\mathrm{P}}^{-}\right\}} \delta \boldsymbol{u}_{J}^{T} \sum_{K \in\left\{\mathcal{W}_{0}^{+}, \mathcal{W}_{\mathrm{P}}^{-}\right\}} \int_{\Omega_{0}^{+} \backslash \Gamma_{0}^{c}} \varrho_{0} N_{J}(\boldsymbol{X})^{T} \cdot N_{K}(\boldsymbol{X}) \mathrm{d} \Omega \ddot{\boldsymbol{u}}_{K} \\
& +\sum_{J \in\left\{\mathcal{W}_{0}^{-}, \mathcal{W}_{\mathrm{P}}^{+}\right\}} \delta \boldsymbol{u}_{J}^{T} \sum_{L \in\left\{\mathcal{W}_{0}^{-}, \mathcal{W}_{\mathrm{P}}^{+}\right\}} \int_{\Omega_{0}^{-} \backslash \Gamma_{0}^{c}} \varrho_{0} N_{J}(\boldsymbol{X})^{T} \cdot N_{L}(\boldsymbol{X}) \mathrm{d} \Omega \ddot{\boldsymbol{u}}_{L} \\
& \delta W_{\mathrm{ext}}=\sum_{I \in\left\{\mathcal{W}_{0}^{+}, \mathcal{W}_{\mathrm{P}}^{-}\right\}} \delta \boldsymbol{u}_{I}^{T}\left[\int_{\Omega_{0}^{+} \backslash \Gamma_{0}^{c}} \varrho_{0} N_{I}(\boldsymbol{X})^{T} \cdot \boldsymbol{b} \mathrm{d} \Omega+\delta \boldsymbol{u}_{I}^{T} \int_{\Gamma_{0}^{t}} N_{I}(\boldsymbol{X})^{T} \cdot \overline{\boldsymbol{t}}_{0} \mathrm{~d} \Gamma\right] \\
& +\sum_{J \in\left\{\mathcal{W}_{0}^{-}, \mathcal{W}_{\mathrm{P}}^{+}\right\}} \delta \boldsymbol{u}_{J}^{T}\left[\int_{\Omega_{0}^{-} \backslash \Gamma_{0}^{c}} \varrho_{0} N_{J}(\boldsymbol{X})^{T} \cdot \boldsymbol{b} \mathrm{d} \Omega+\int_{\Gamma_{0}^{t}} N_{J}(\boldsymbol{X})^{T} \cdot \overline{\boldsymbol{t}}_{0} \mathrm{~d} \Gamma\right] \\
& \delta W_{\mathrm{coh}}=\sum_{I \in\left\{\mathcal{W}_{0}^{+}, \mathcal{W}_{\mathrm{P}}^{-}\right\}} \delta \boldsymbol{u}_{I}^{T} \int_{\Gamma_{0}^{c}} N_{I}(\boldsymbol{X})^{T} \cdot \overline{\boldsymbol{t}}_{c} \mathrm{~d} \Gamma \\
& -\sum_{J \in\left\{\mathcal{W}_{0}^{-}, \mathcal{W}_{\mathrm{P}}^{+}\right\}} \delta \boldsymbol{u}_{J}^{T} \int_{\Gamma_{0}^{c}} N_{J}(\boldsymbol{X})^{T} \cdot \overline{\boldsymbol{t}}_{c} \mathrm{~d} \Gamma
\end{aligned}
$$

Using the fundamental lemma of the variational principle, we obtain the discretized equation, i.e.

$$
\mathbf{M} \ddot{\mathbf{q}}=\mathbf{f}_{\mathrm{ext}}+\mathbf{f}_{\mathrm{coh}}-\mathbf{f}_{\mathrm{int}}
$$

where $\mathbf{M}$ is the consistent mass matrix, $\mathbf{q}$ are the generalized parameters, $\mathbf{f}_{\text {ext }}, \mathbf{f}_{\text {int }}$ and $\mathbf{f}_{\text {coh }}$ are the discrete external, internal and cohesive force vectors, respectively. The expressions for $\mathbf{M}$, 
$\mathbf{q}, \mathbf{f}_{\text {ext }}, \mathbf{f}_{\mathrm{coh}}$ and $\mathbf{f}_{\mathrm{coh}}$ are given by

$$
\begin{aligned}
\mathbf{M} & =\int_{\Omega_{0} \backslash \Gamma_{0}^{c}} \varrho_{0} \mathbf{N}^{0^{T}} \mathbf{N}^{0} \mathrm{~d} \Omega \\
\mathbf{f}_{\text {int }} & =\int_{\Omega_{0} \backslash \Gamma_{0}^{c}} \mathbf{B}^{0^{T}} \mathbf{P} \mathrm{d} \Omega \\
\mathbf{f}_{\mathrm{ext}} & =\int_{\Omega_{0} \backslash \Gamma_{0}^{c}} \varrho_{0} \mathbf{N}^{0^{T}} \mathbf{b} \mathrm{d} \Omega+\int_{\Gamma_{0}^{t}} \mathbf{N}^{0^{T}} \overline{\mathbf{t}}_{0} \mathrm{~d} \Gamma \\
\mathbf{f}_{\mathrm{coh}} & =\int_{\Gamma_{0}^{c}} \mathbf{N}^{0^{T}} \mathbf{t}_{c 0} \mathrm{~d} \Gamma_{0} \\
\mathbf{q} & =\left[\boldsymbol{u}_{I} \boldsymbol{u}_{J}\right]^{T} \quad \forall I \in\left\{\mathcal{W}_{0}^{+}, \mathcal{W}_{\mathrm{P}}^{-}\right\} \text {and } J \in\left\{\mathcal{W}_{0}^{-}, \mathcal{W}_{\mathrm{P}}^{+}\right\} \\
\mathbf{N}^{0} & =\left[N_{I} N_{J}\right] \quad \forall I \in\left\{\mathcal{W}_{0}^{+}, \mathcal{W}_{\mathrm{P}}^{-}\right\} \text {and } J \in\left\{\mathcal{W}_{0}^{-}, \mathcal{W}_{\mathrm{P}}^{+}\right\} \\
\mathbf{B}^{0} & =\nabla_{0} \mathbf{N}^{0}
\end{aligned}
$$

We used this approach in combination with the Belytschko and Bindemann [10]'s element for quadrilateral elements.

\section{NUMERICAL EXAMPLES AND DISCUSSION}

\subsection{Edge crack}

This example is to study the robustness of our element when the crack tip is very close to a node or an element side. Consider the edge crack problem as shown in figure 8 with $\sigma=1$, Young's modulus $E=100$, Poisson's ratio $\nu=0.3$ and the ratio $a / W=0.05$. In the analytical solution, the stress intensity factor for this problem is $K_{I}=1.253$. Since it is easier to control the distance of the crack to a node or an edge for structured meshes, we study our 4-node quadrilateral element. The results will be similar for the triangular elements.

Figure 9 shows the stress intensity factor with increasing mesh density for the triangular and quadrilateral element. Here, the position of the crack tip within an element varies arbitrarily. However, this does not influence the convergence rate. For approximately 18,000 elements, we modified the position of the crack tip within the element by locally rearranging some elements, figure 8 and table I. Note that if a crack is too close to an edge it will be closed at the element side as stated earlier. Table I shows the influence of the stress intensity factors for different positions of the crack tip in the element. The differences in the results are marginal, i.e. less than $6 \%$ deviation.

\subsection{Double-cantilever-beam test with diagonal loads}

This example was chosen to demonstrate the advantages of our new element with respect to the standard phantom-node element without tip enrichment. Therefore consider the doublecantilever beam (DCB) with diagonal load as shown in Fig. 10. The diagonal load $F_{2}(t)$ is increased up to a certain pseudo time proportional to the tensile load $F_{1}(t)$. Then, load $F_{2}$ is kept constant while $F_{1}$ is still increased. More details can be found in Kobayashi et al. [27]. A straight crack occurred in the experiment traveling in a $71^{\circ}$ against the horizontal axis.

Int. J. Numer. Meth. Engng 2000; 00:1-6 


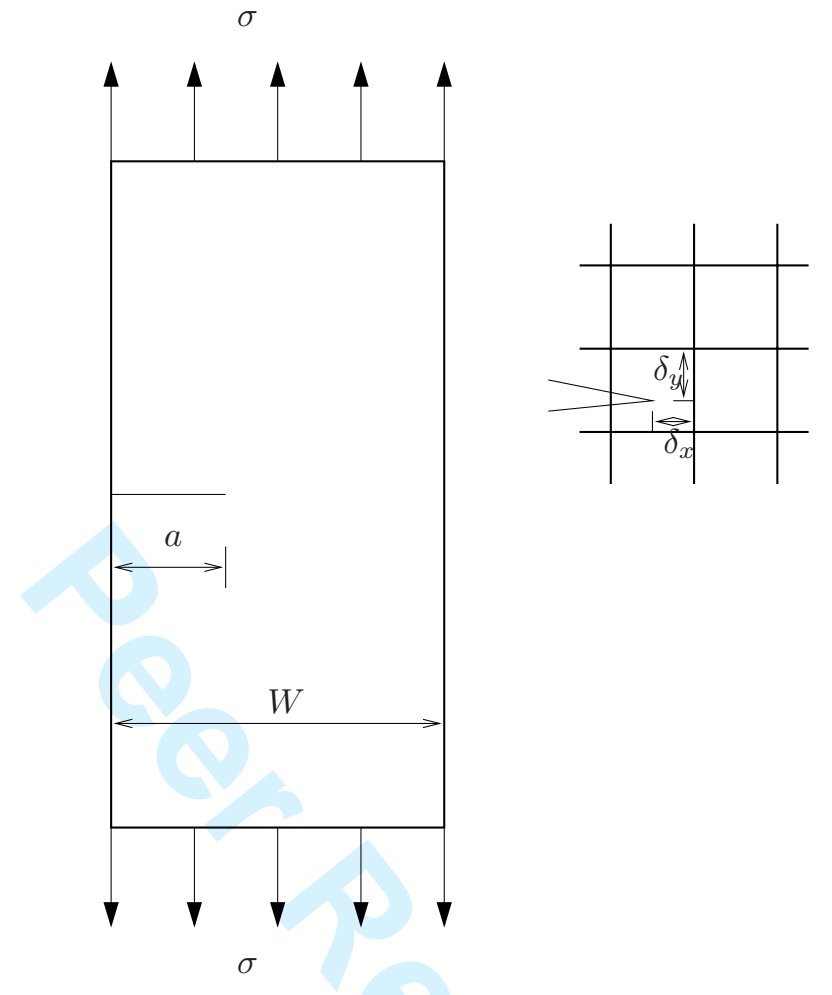

Figure 8. The edge crack problem.

Table I. Normalized SIF values $K_{I}$ for different positions of the crack tip for a structured discretization with 18,000 elements.

\begin{tabular}{c|c|c|c|c}
\hline$\delta_{x} / h$ & 0.05 & 0.1 & 0.2 & 0.3 \\
$\delta_{y} / h$ & & & & \\
\hline 0.1 & 0.9184 & 0.9212 & 0.9254 & 0.927 \\
0.2 & 0.9233 & 0.9255 & 0.926 & 0.9268 \\
0.3 & 0.9222 & 0.926 & 0.926 & 0.9269 \\
\hline
\end{tabular}

The material parameters are Young's modulus $E=30,500 \mathrm{MPa}$, Poisson's ratio $\nu=0.2$, tensile strength $f_{t}=3 \mathrm{MPa}$ and fracture energy $G_{f}=100 \mathrm{~N} / \mathrm{m}$.

We have discretized the specimen with different meshes of different refinements, starting with approximately 100 elements up to 7,000 elements, figure 11 . We will show only results for the unstructured meshes though the results for the structured meshes were similar.

Fig. 12 shows the load $F_{1}$ crack opening plot, see Fig. 11d for the displacement plot and for definitions. In the conventional phantom node method, the crack has to be propagated through the entire element that leads to very rough load deflection curves and inaccurate results for the coarse meshes. For our crack tip enrichment, we control the crack length mainly 


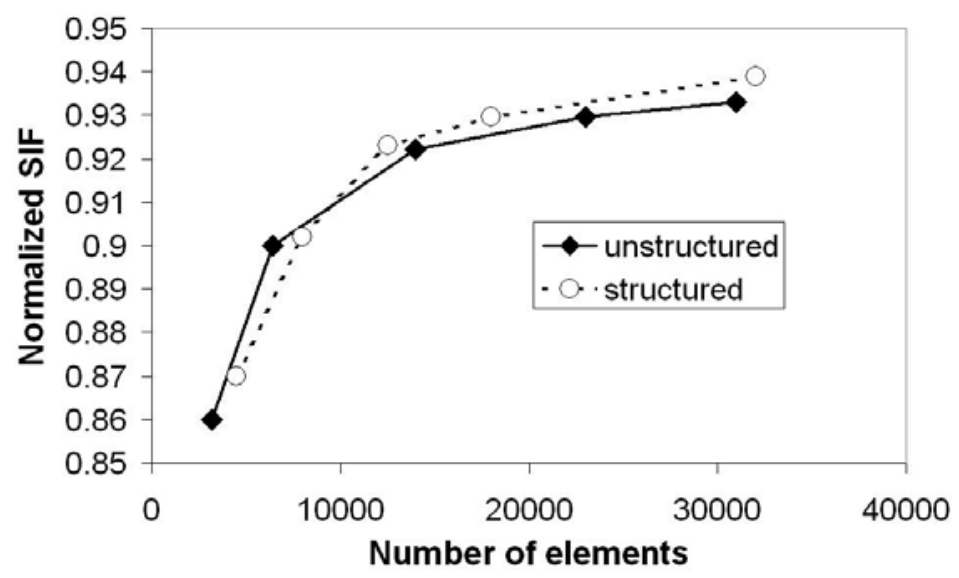

Figure 9. Normalized stress intensity factor for the edge crack problem for structured and unstructured meshes with different mesh sizes.

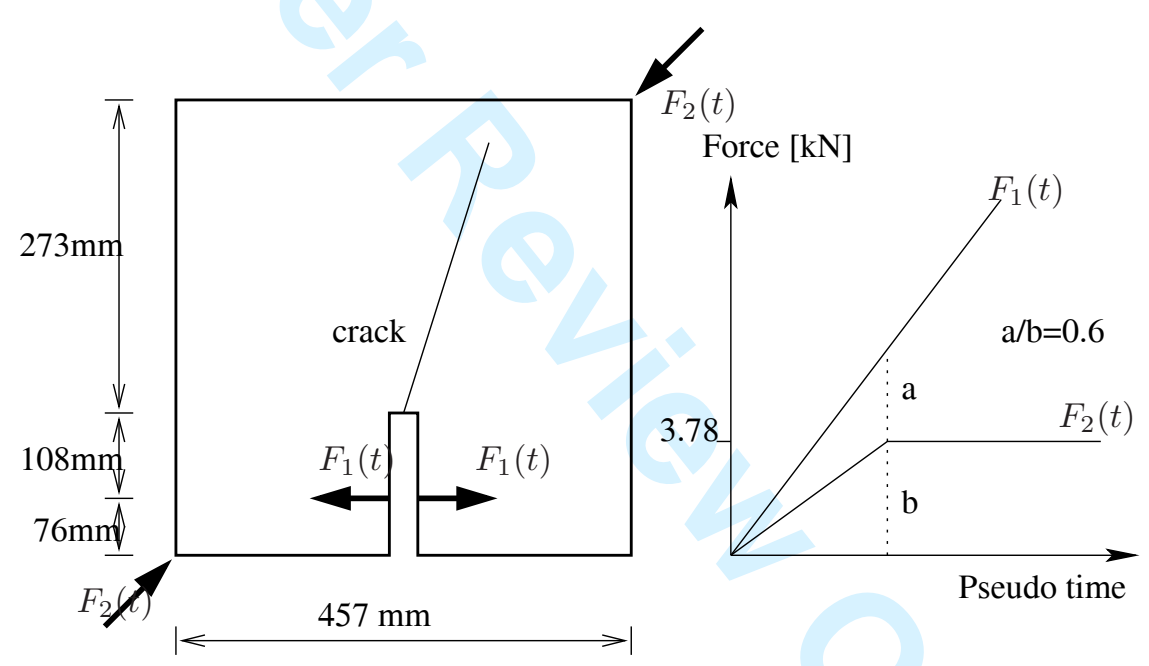

Figure 10. The tensile/shear beam from Kobayashi et al. [27].

for convergence reasons and keep for all simulations a constant crack length of $3 \mathrm{~mm}$. The load deflection curve for our tip element included in Fig. 12 does not show the rough behavior of the conventional phantom-node method.

\subsection{Arrea-Ingraffea beam}

Consider the Arrea and Ingraffea [3] beam that fails in a combined tensile-shear mode. The beam is loaded at points A and B according to Fig. 13. The Young's modulus is 28,000 MPa, 


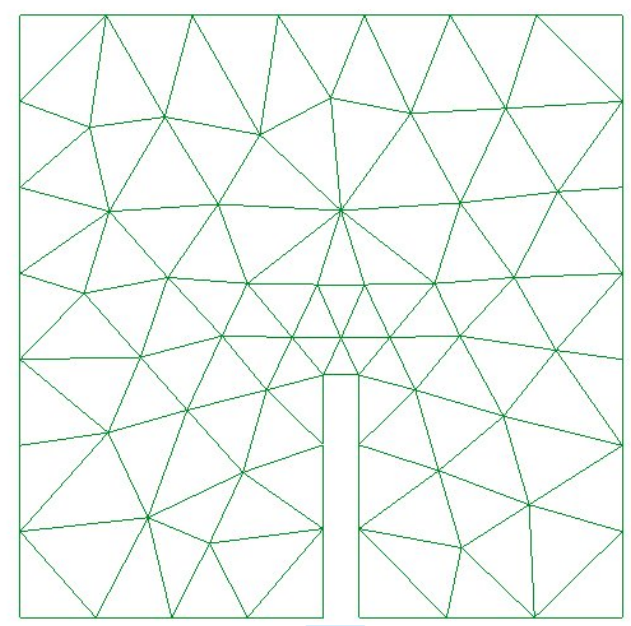

(a)

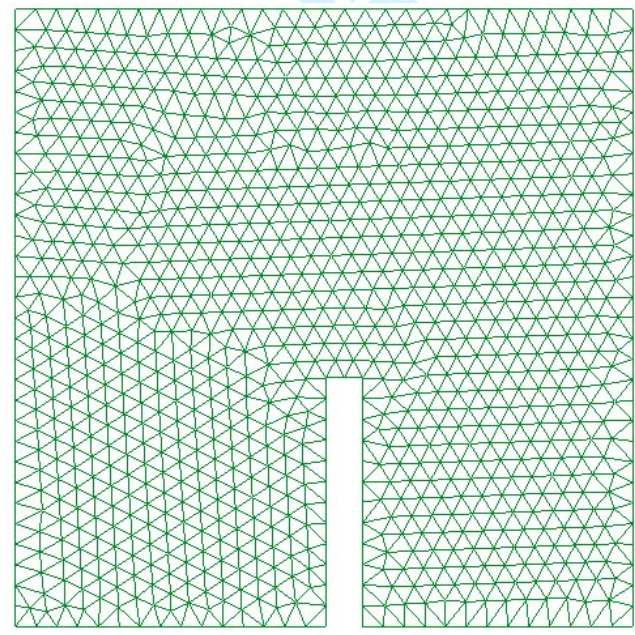

(c)

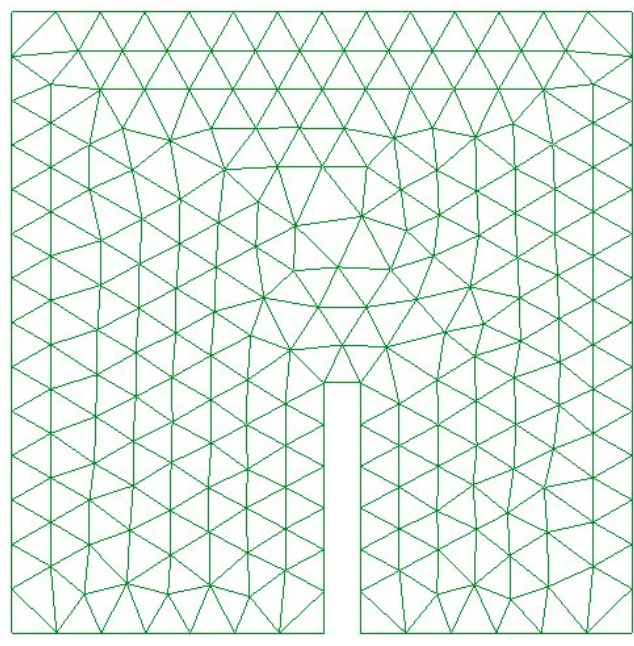

(b)

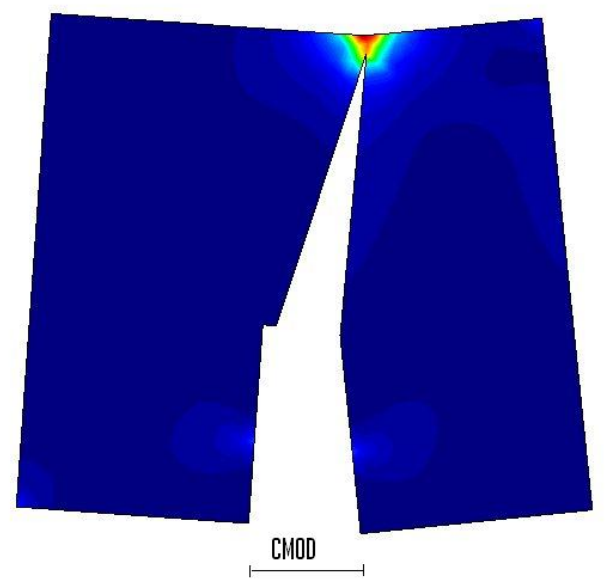

(d)

Figure 11. Different meshes for the DCB problem and crack pattern at the end of the computation

tensile strength is $2.8 \mathrm{MPa}$ and Poisson's ration $\nu=0.18$. We consider a Rankine material with linear decaying cohesive law and a fracture energy $G_{f}=100 \mathrm{~N} / \mathrm{m}$. We tested different mesh refinements starting from 400 elements up to 12,000 elements and compared our results to the original phantom node method where the crack is introduced through the entire element.

The final crack path is shown in Fig. 14, exemplarily for 2,800 triangular elements, as well as certain stress distributions in the beam. As can be seen, the crack curves nicely that is also observed experimentally. The crack pattern for the quadrilateral meshes are similar. The load displacement curve (crack mouth sliding right of the notch) is shown in Fig. 15a,b for different 


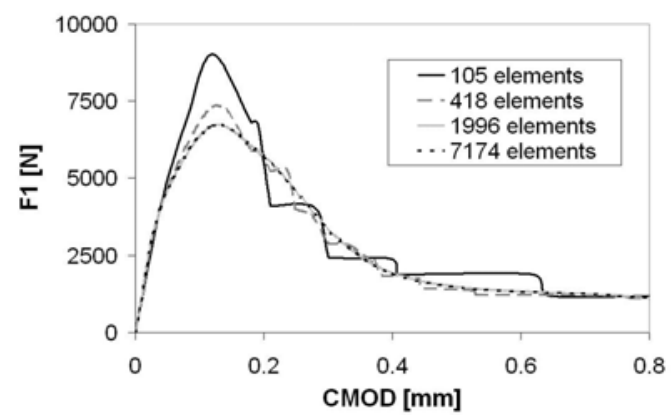

(a) conventional phantom node method

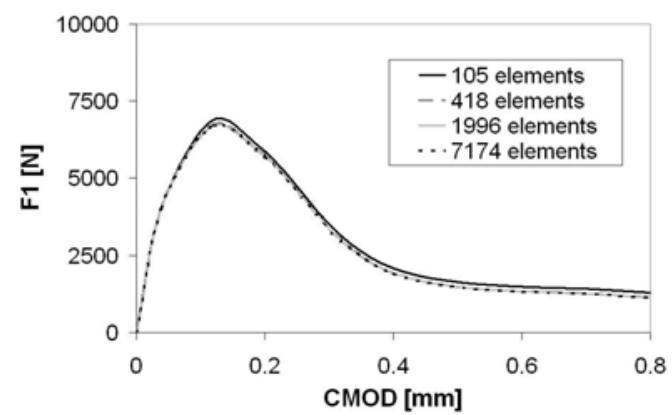

(b) tip enriched phantom node method

Figure 12. Load-displacement (CMOD) curve for the DCB problem for the conventional phantom-node method and with tip enriched phantom-node method

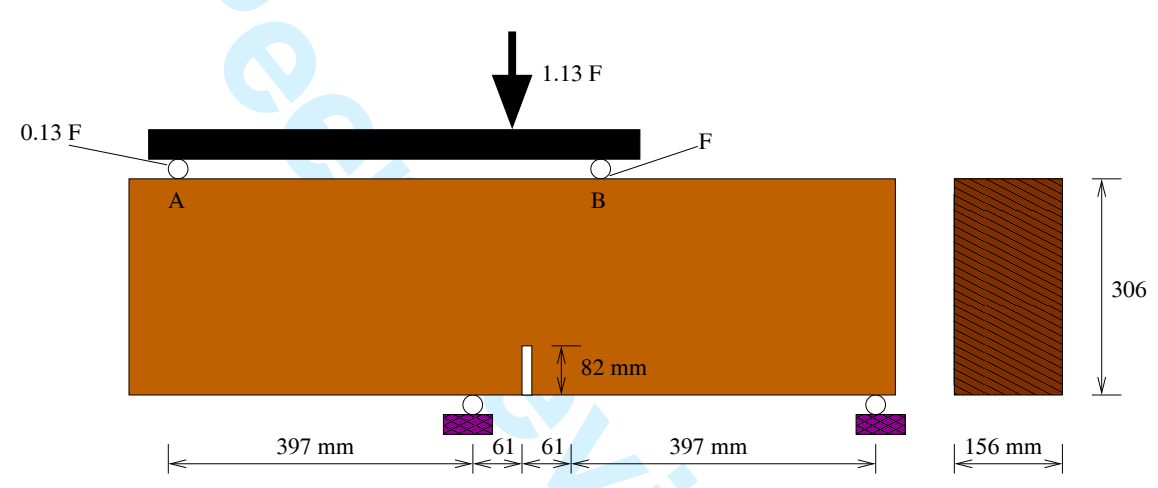

Figure 13. The tensile/shear beam from Arrea Ingraffea

meshes $^{\dagger}$ and lies in the experimental scatter. The results of the conventional phantom node method is shown in figure $15 \mathrm{c}$ for the same unstructured discretizations and the load deflection curves are rougher especially for the coarse meshes.

\subsection{Crack branching}

Let us consider a rectangular prenotched specimen as shown in Fig. 16. The length of the specimen is $0.1 \mathrm{~m}$ and the width $0.04 \mathrm{~m}$. Plane strain conditions are assumed. There is a horizontal initial-crack from the left edge to the center of the plate over the entire thickness. On the top and bottom edges, tensile traction of $1 \mathrm{MPa}$ is applied.

We used the Rankine criterion and an exponential decaying cohesive law (Fig. 7)c. The material parameters were Young's modulus $E=32 \mathrm{MPa}$, Poisson's ratio $\nu=0.2$ and density

\footnotetext{
$\dagger^{\dagger}$ triangular versus quadrilateral and also different refinements
} 


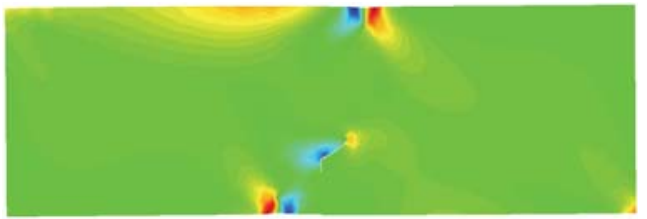

(a)

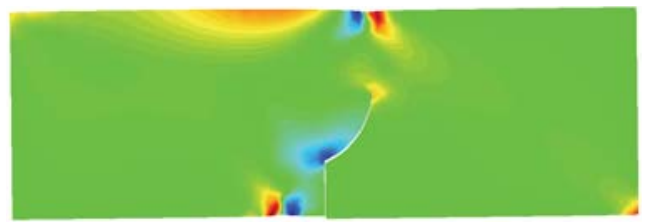

(c)

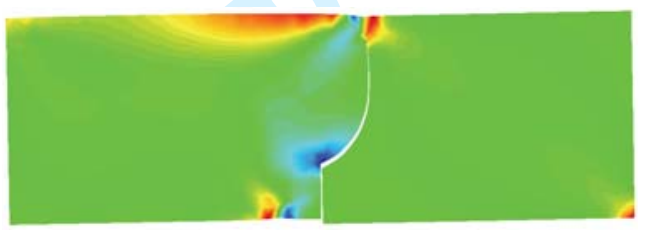

(e)

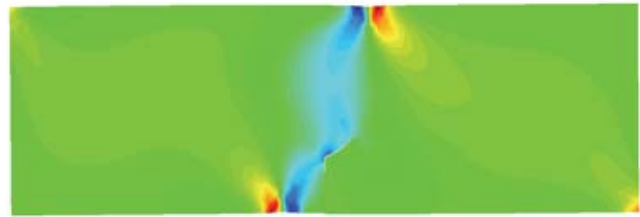

(b)

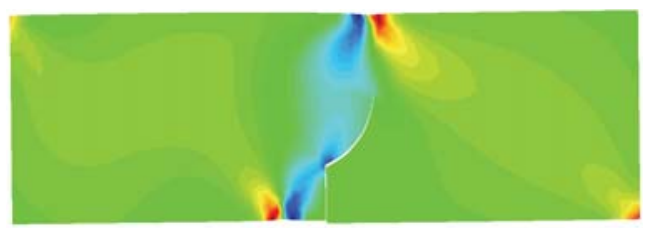

(d)

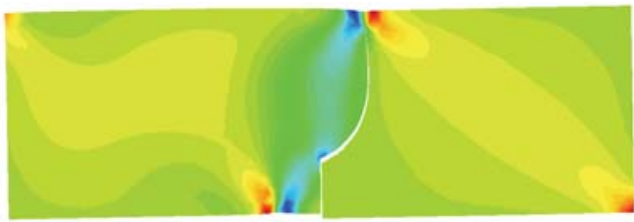

(f)

Figure 14. The crack pattern and stress distribution for the Arrea-Ingraffea beam at different load steps; (a,c,e) principal tensile stress and (e,f,g) shear stress.

$\varrho=2,450 \mathrm{~kg} / \mathrm{m}^{3}$. Two dimensional computations of this problem had previously been reported by Belytschko et al. [16], Falk et al. [21], Rabczuk and Belytschko [38], Xu and Needleman [51]; and three dimensional computations e.g. by Rabczuk and Belytschko [39]. Experimental data is available; see Fineberg et al. [22], Ravi-Chandar [42], Sharon and Fineberg [43].

We carried out simulations with both the quadrilateral elements and the triangular elements. We tested meshes consisting of 1,000 and 16,000 of the quadrilateral elements and 2,000 and 10,000 of the triangular elements. The crack pattern of the specimen at different times in the deformed configuration is shown in Fig. 17. Note that the deformations are shown magnified. Stress distributions in the specimen at different time steps are shown in Fig. 18.

The time history of the crack speed is shown in Fig. 19. The crack starts to propagate at about $0.012 \mathrm{~ms}$. As expected, the crack speed is highest at the time of crack branching. In order to model crack branching, we used the conventional phantom node method since the point of branching cannot be described by our proposed method. At the first branching, the crack speed almost reaches the theoretical Rayleigh wave speed. Afterwards, the crack speed decreases and the crack speed of the upper branch is shown. The crack speed of the lower branch is very similar as Fig. 19 might indicate.

\subsection{Kalthoff and Winkler [26]'s experiment}

Kalthoff and Winkler [26] performed a series of experiments in which a steel plate was hit by a projectile with different impact velocities as shown in Fig. 20. They discovered different failure 


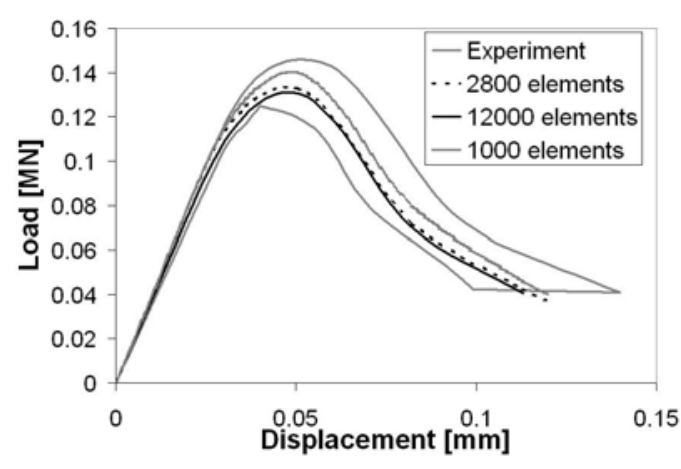

a)

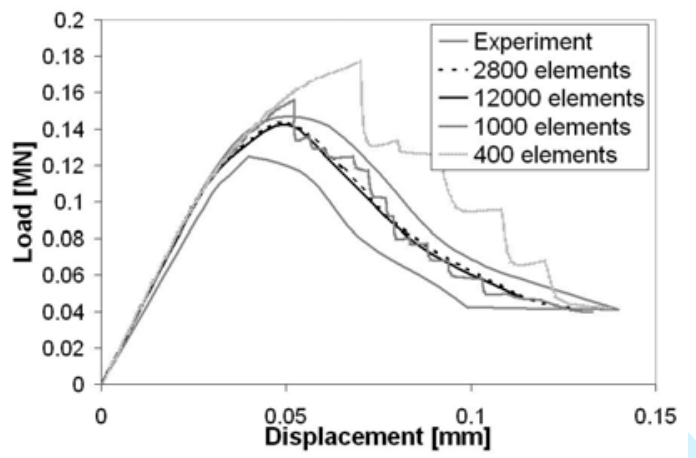

c)

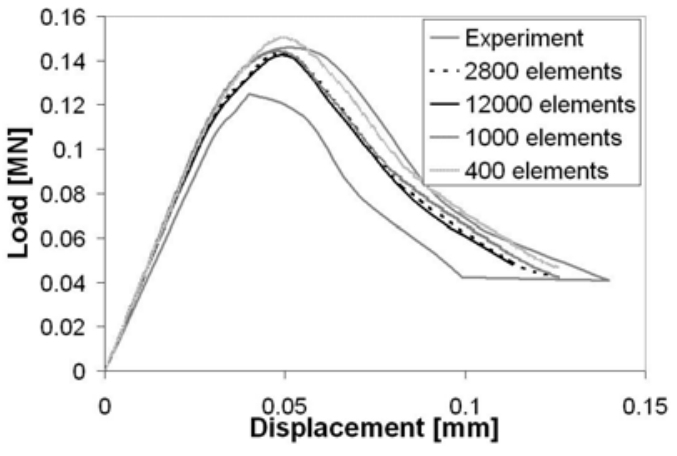

b)

Figure 15. Load-deflection curve of the tensile/shear beam from [3] for (a) structured tip-enriched phantom node discretization, (b) unstructured tip-enriched phantom node discretization and (c) unstructured conventional phantom node discretization

phenomena for different impact velocities. Up to an impact velocity of about $v_{c}=20 \mathrm{~m} / \mathrm{s}$, the steel plate fails brittle and a crack develops in a $70^{\circ}$ against the axis parallel to the flight direction of the projectile. For larger impact velocities, a ductile failure pattern was observed and a shear band develops from the onset of the notch in a much flatter angle of the opposite direction. We also note that the transition from brittle to ductile failure depends on the size and shape of the notch. We focus on the brittle failure pattern and an impact velocity of 17 $\mathrm{m} / \mathrm{s}$. The simulation of this problem had been reported by Belytschko et al. [16], Menouillard et al. [29], Rabczuk et al. [41], Xu and Needleman [51], etc.

We used structured and unstructured discretizations with three different mesh-refinements, for the structured case between approximately 2,000 and 16,000 elements and for the unstructured case between 1,000 and 10,000 elements. The Johnson Cook model [25] was used with material parameters: $A=792 \mathrm{MPa}, B=509 \mathrm{MPa}, C=0.014, n=0.26, m=0.55$, $\rho=7,800 \mathrm{~kg} / \mathrm{m}^{3}, T_{0}=293 \mathrm{~K}, K=157 \mathrm{GPa}, G=76 \mathrm{GPa}, c_{v}=477 \mathrm{~J} / \mathrm{kg}^{\circ} \mathrm{C}, T_{r}=296$ $\mathrm{K}, T_{m}=1,033 \mathrm{~K}, \gamma_{0}=1.3 \times 10^{-13} \mathrm{~s}^{-1}$ and $\kappa_{0}=500 \mathrm{~K}$. An exponential cohesive law was used. We exploited the symmetry and modeled only half of the specimen. The crack pattern and the stress distribution at two different time steps is shown in Fig. 21. The crack speed for 


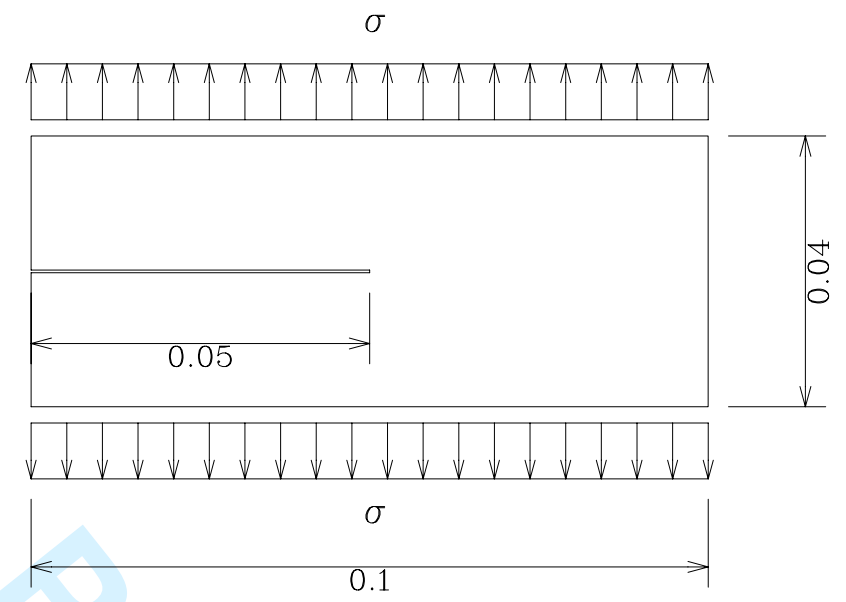

Figure 16. A plate with an edge crack loaded by uniform traction on the top and bottom edges.

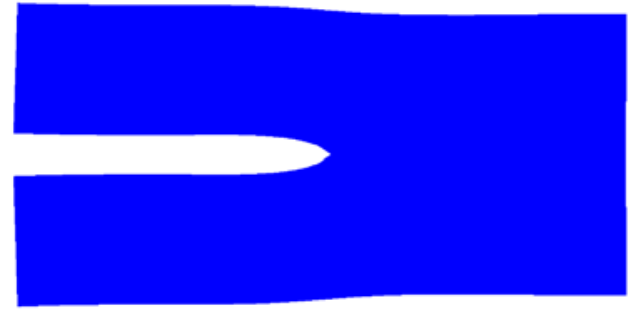

(a)

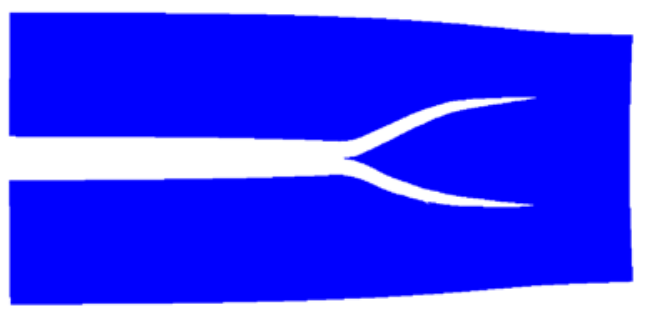

(c)

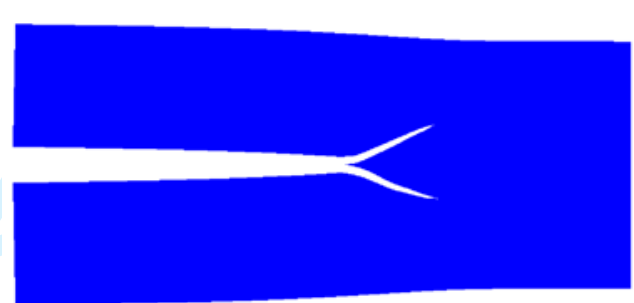

(b)

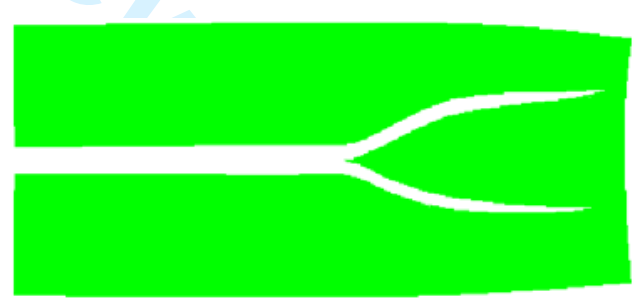

(d)

Figure 17. The crack configuration in the deformed configuration of the crack branching problem at different times

different simulations is illustrated in Fig. 22. The crack path agrees well with the experimental observation. 


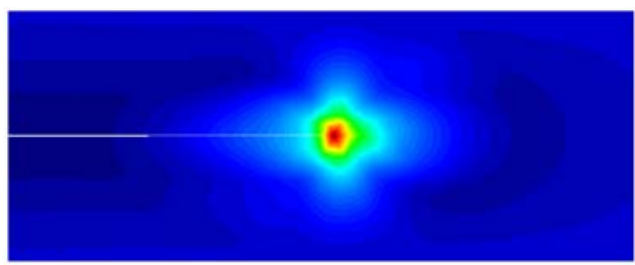

(a)

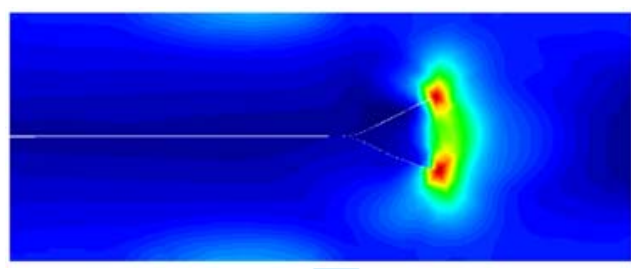

(c)

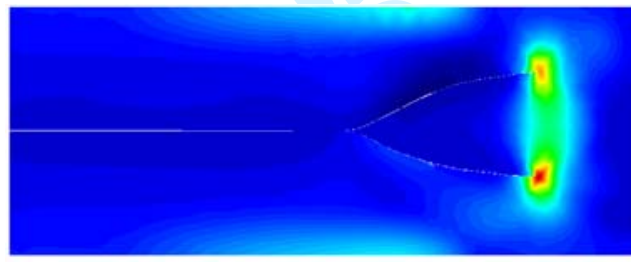

(e)

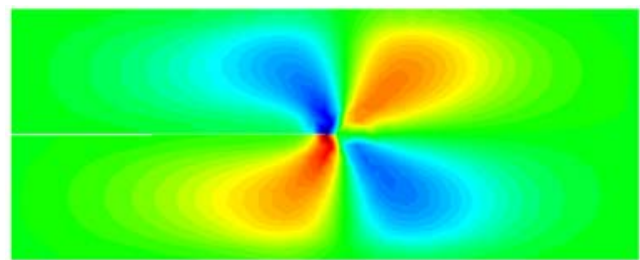

(b)

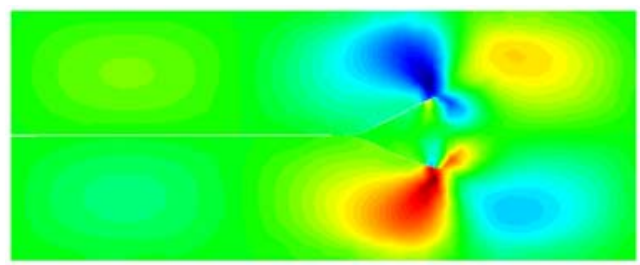

(d)

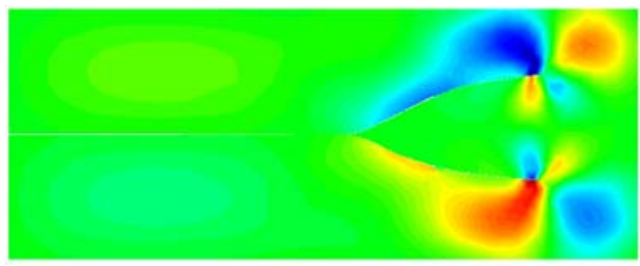

(f)

Figure 18. The crack pattern and stress distribution for the branching problem at different load steps; (a,c,e) principal tensile stress and (e,f,g) shear stress.

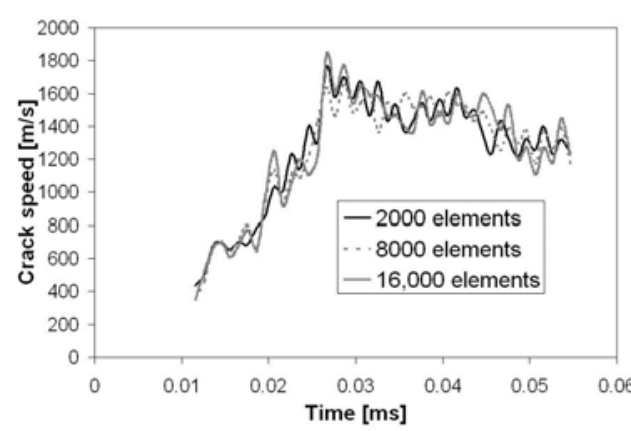

(a)

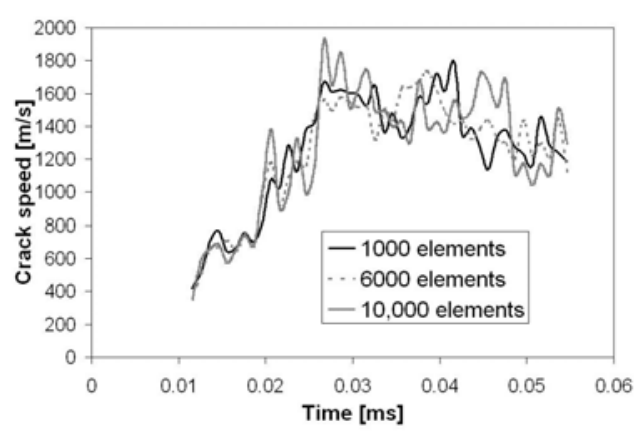

(b)

Figure 19. The crack speed time history of the crack branching problem for different mesh refinements; (a) the quadrilateral elements and (b) the triangular elements. 


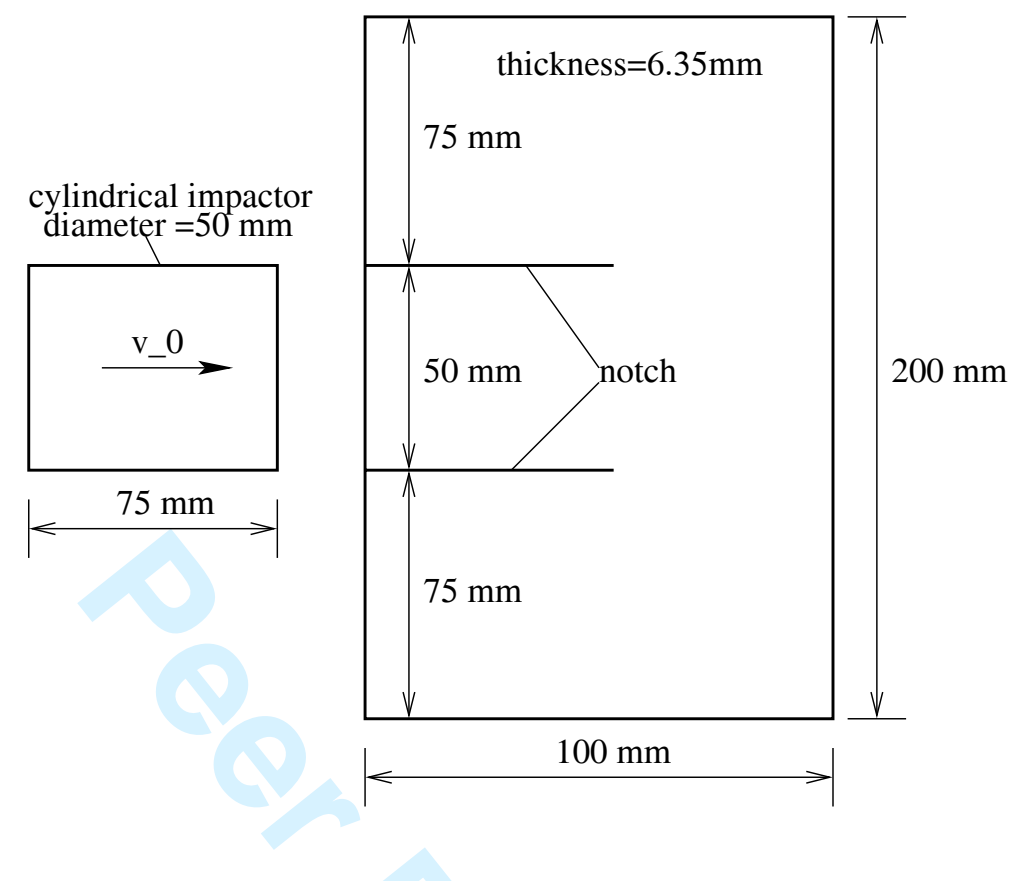

Figure 20. The Kalthoff problem: test setup

\section{CONCLUSIONS}

1. We presented an extension of the phantom node method [44] by allowing crack tips to be placed within a finite element. Therefore the crack growth in the phantom node method became almost independent of the finite element mesh.

2. The formulation for the crack tip was developed for a three node triangular element and a four node quadrilateral element. It may be possible to extend this approach to a three dimensional element such as a four node tetrahedron element.

3. Due to the lack of an enrichment, techniques such as EAS can easily be implemented into the formulation.

4. For a simple example with available analytical solution, we have studied the method for several positions of the crack tip within an element in order to study the reliability and accuracy of the method and found the method robust. The conventional phantom node method is used if the crack tip is too close to an edge or a node.

5. This method was especially useful for dynamic fracture problems when it was used with the one point quadrature rule.

6. Cohesive zone models were used in the postlocalization region to guarantee the wellposedness of the IBVP. We employed this method to several quasi-static and dynamic crack problems and compared our results to experimental data or other numerical results in the literature and found good agreement.

7. We have described the crack segments explicitly by piecewise linear segments and mostly controlled the crack length. However, when level sets are used for the representation of the

Int. J. Numer. Meth. Engng 2000; 00:1-6 


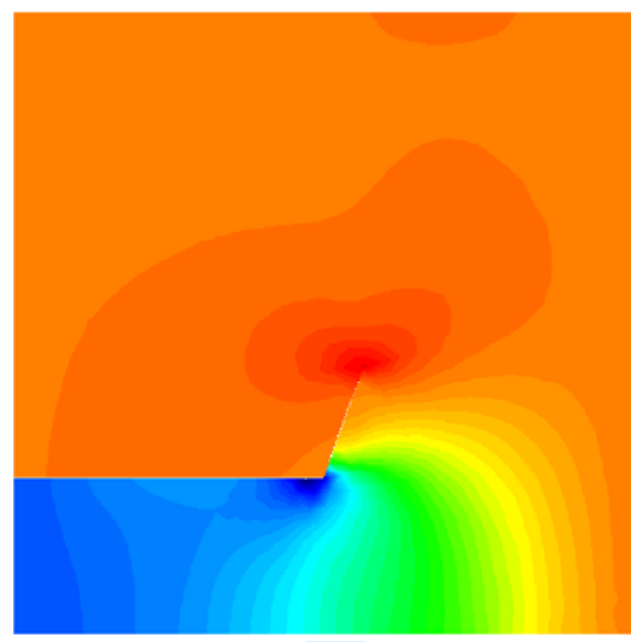

(a) $\sigma_{x x}$

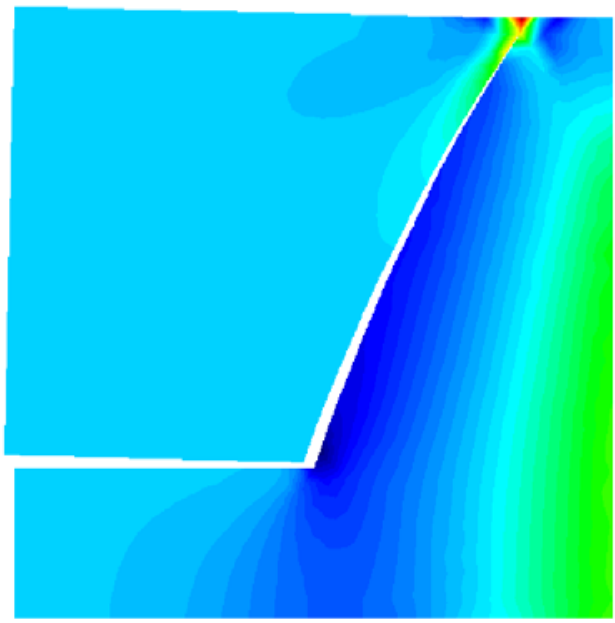

(c) $\sigma_{y y}$

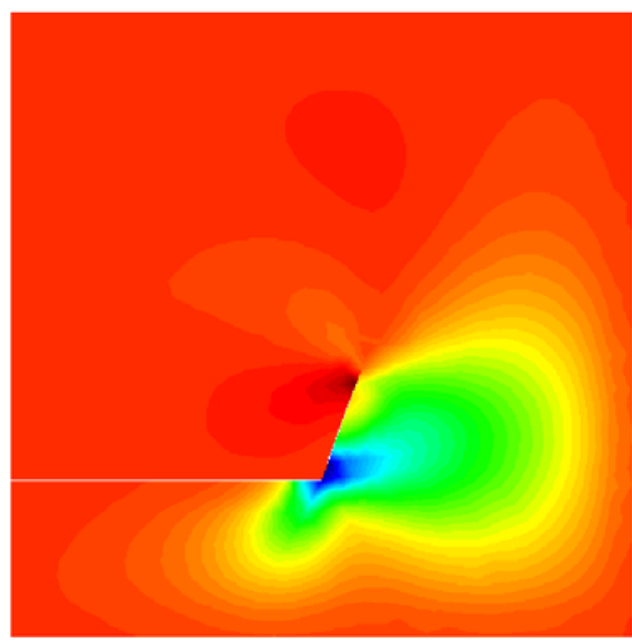

(b) $\sigma_{x y}$

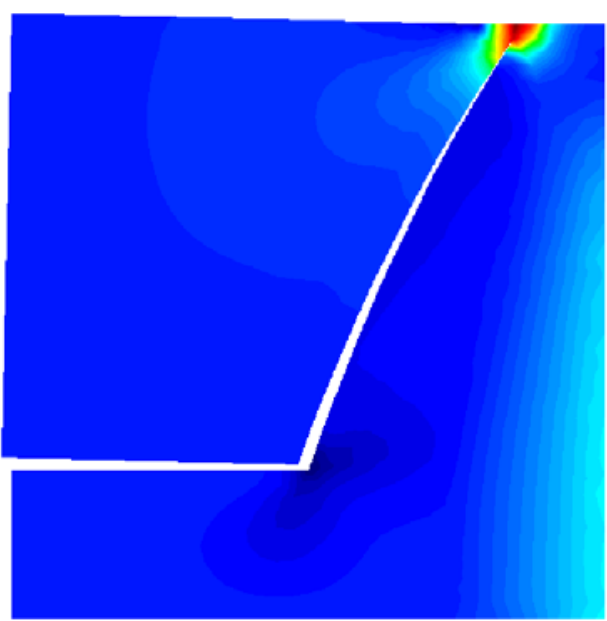

(d) $\sigma_{1}$

Figure 21. The stress distribution and crack pattern in the Kalthoff experiment at different times

crack, the crack speed and hence the crack length can be obtained via the Hamiltonian Jacobi equation solving with respect to the crack speed as shown in Belytschko et al. [16]. For that case, the opportunity to close the crack within a finite element is advantageous.

8. We employed the method to several quasi-static and dynamic crack problems and compared our results to experimental data or other numerical results in the literature and found good agreement. 


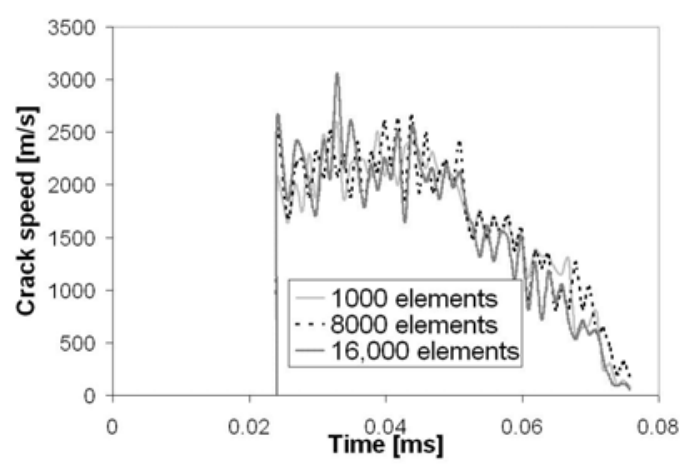

(a)

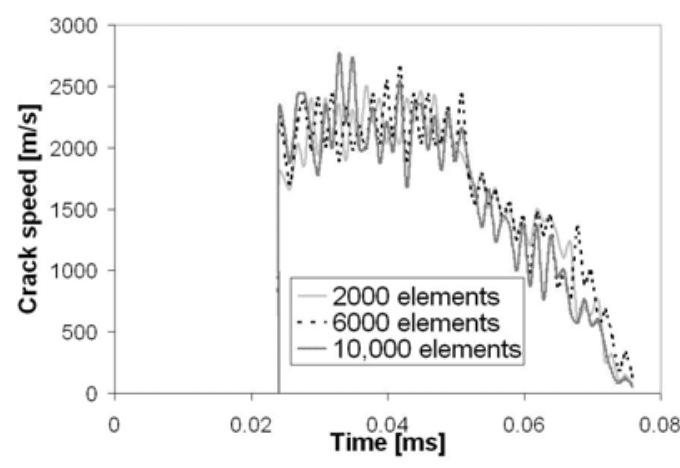

(b)

Figure 22. The crack speed for the Kalthoff problems for different mesh refinements for (a) the quadrilateral elements and (b) the triangular elements

references

[1] P.M.A. Areias and T. Belytschko. Analysis of three-dimensional crack initiation and propagation using the extended finite element method. International Journal for Numerical Methods in Engineering, 63:760-788, 2005.

[2] P.M.A. Areias, J.H. Song, and T. Belytschko. Analysis of fracture in thin shells by overlapping paired elements. International Journal for Numerical Methods in Engineering, 195:5343-5360, 2006.

[3] M. Arrea and A.R. Ingraffea. Mixed-mode crack propagation in mortar and concrete. Technical Report 81-13, Dept. of Struct. Eng., Cornell University Ithaka, 1982.

[4] R. C. Batra and R. R. Gummalla. Effect of material and geometric parameters on deformations near the notch-tip of a dynamically loaded prenotched plate. International Journal of Fracture, 101(1-2):99-140, 2000.

[5] R.C. Batra and N.A. Jaber. Failure mode transition in an impact loaded pre-notched plate with four thermoviscoplastic relations. International Journal of fracture, 110:47-71, 2001.

[6] R.C. Batra and M.H. Lear. Simulation of brittle and ductile fracture in an impact loaded prenotched plate. International Journal of Fracture, 126:179-203, 2004.

[7] R.C. Batra and N.V. Nechitalio. Analysis of failure modes in impulsively loaded prenotched plates. International Journal of plasticity, 13:291-308, 1997.

[8] R.C. Batra, N.A. Jaber, and M.E. Malsbury. Analysis of failure modes in an impact loaded thermoviscoplastic prenotched plate. International Journal of plasticity, 19:139$196,2003$. 
[9] Z. P. Bažant and F. C. Caner. Microplane model M5 with kinematic and static constraints for concrete fracture and anelasticity. I: Theory. Journal of Engineering Mechanics, ASCE, 131(1):31-40, 2005.

[10] T. Belytschko and L.P. Bindemann. Assumed strain stabilization of the 4-node quadrilateral with 1-point quadrature for nonlinear problems. Computer Methods in Applied Mechanics and Engineering, 88:311-340, 1991.

[11] T. Belytschko and T. Black. Elastic crack growth in finite elements with minimal remeshing. International Journal for Numerical Methods in Engineering, 45(5):601-620, 1999.

[12] T. Belytschko and Y.Y. Lu. Element-free galerkin methods for static and dynamic fracture. International Journal of Solids and Structures, 32:2547-2570, 1995.

[13] T. Belytschko, Y.Y. Lu, and L. Gu. Crack propagation by element-free galerkin methods. Engineering Fracture Mechanics, 51(2):295-315, 1995.

[14] T. Belytschko, D. Organ, and M. Tabbara. Numerical simulations of mixed mode dynamic fracture in concrete using element-free Galerkin methods. ICES conference proceedings, 1995.

[15] T. Belytschko, N. Moës, S. Usui, and C. Parimi. Arbitrary discontinuities in finite elements. International Journal for Numerical Methods in Engineering, 50(4):993-1013, 2001.

[16] T. Belytschko, H. Chen, J. Xu, and G. Zi. Dynamic crack propagation based on loss of hyperbolicity with a new discontinuous enrichment. International Journal for Numerical Methods in Engineering, 58(12):1873-1905, 2003.

[17] S. Bordas and B. Moran. Extended finite element and level set method for damage tolerance assessment of complex structures: an object-oriented approach. EFM, 2006. in press.

[18] G. Camacho and M. Ortiz. Computational modelling of impact damage in brittle materials. International Journal of Solids and Structures, 33:2899-2938, 1996.

[19] J. Chessa, H. Wang, and T. Belytschko. On the construction of blending elements for local partition of unity enriched finite elements. International Journal for Numerical Methods in Engineering, 57(7):1015-1038, 2003.

[20] J. Dolbow, N. Moes, and T. Belytschko. Discontinuous enrichment in finite elements with a partition of unity method. Finite Element Analysis and Design, 36(3):235-260, 2000.

[21] M. L. Falk, A. Needleman, and J. R. Rice. A critical evaluation of cohesive zone models of dynamic fracture. Journal de Physique IV, 11(PR5):43-50, 2001.

[22] J. Fineberg, E. Sharon, and G. Cohen. Crack front waves in dynamic fracture. International Journal of Fracture, 121(1-2):55-69, 2003.

Int. J. Numer. Meth. Engng 2000; 00:1-6 
[23] Ji H., Chopp D., and Dolbow J. E. A hybrid finite element/level set method for modelling phase transformation. International Journal for Numerical Methods in Engineering, 54 (8):1209-1233, 2002.

[24] A. Hansbo and P. Hansbo. A finite element method for the simulation of strong and weak discontinuities in solid mechanics. Computer Methods in Applied Mechanics and Engineering, 193(33-35):3523-3540, 2004.

[25] G. R. Johnson and W. H. Cook. A constitutive model and data for metals subjected to large strains, high strain rates, and high temperatures. In Proceedings of 7 th International Symposium on Ballistics, 1983.

[26] J. F. Kalthoff and S. Winkler. Failure mode transitions at high rates of shear loading. In C. Y. Chiem, H.-D. Kunze, and L. W. Meyer, editors, Impact Loading and Dynamic Behaviour of Materials, volume 1, pages 185-195. Informationsgesellschaft, Verlag, 1988.

[27] A. S. Kobayashi, A. F. Emery, and S. Mall. Dynamic-finite-element and dynamicphotoelastic analyses of two fracturing homalite-100 plates. Experimental Mechanics, 16:321-328, 1976.

[28] A. Legay, J. Chessa, and T. Belytschko. An eulerianlagrangian method for fluidstructure interaction based on level sets. Computer Methods in Applied Mechanics and Engineering, 95(17-18):2070-2087, 2006.

[29] T. Menouillard, J. Réthoré, A. Combescure, and H. Bung. Efficient explicit time stepping for the extended finite element method (X-FEM). International Journal for Numerical Methods in Engineering, 68(9):911-939, 2006.

[30] T. Menouillard, J. Réthoré, N. Moës, A. Combescure, and H. Bung. Mass lumping strategies for X-FEM explicit dynamics: Application to crack propagation. International Journal for Numerical Methods in Engineering, 2007.

[31] J. Mergheim, E. Kuhl, and P. Steinmann. A finite element method for the computational modelling of cohesive cracks. International Journal for Numerical Methods in Engineering, 63:276-289, 2005.

[32] J. Mergheim, E. Kuhl, and P. Steinmann. Towards the algorithmic treatment of 3d strong discontinuities. Communications in Numerical Methods in Engineering, 23:97-108, 2007.

[33] N Moes and T. Belytschko. Extended finite element method for cohesive crack growth. Engineering Fracture Mechanics, 69:813-834, 2002.

[34] N. Moës, J. Dolbow, and T. Belytschko. A finite element method for crack growth without remeshing. International Journal for Numerical Methods in Engineering, 46(1):131-150, 1999.

[35] N. Moes, J. Dolbow, and T. Belytschko. A finite element method for crack growth without remeshing. International Journal for Numerical Methods in Engineering, 46(1):133-150, 1999.

Int. J. Numer. Meth. Engng 2000; 00:1-6 
[36] D. Organ, M. Fleming, T. Terry, and T. Belytschko. Continuous meshless approximations for nonconvex bodies by diffraction and transparency. Computational Mechanics, 18:225$235,1996$.

[37] A. Pandolfi, P. R. Guduru, and M. Ortiz. Three dimensional cohesive-element analysis and experiments of dynamic fracture in C300 steel. International Journal of Solids and Structures, 37:3733-3760, 2000.

[38] T. Rabczuk and T. Belytschko. Cracking paricles: A simplified meshfree method for arbitrary evolving cracks. International Journal for Numerical Methods in Engineering, 61:2316-2343, 2004.

[39] T. Rabczuk and T. Belytschko. A three dimensional large deformation meshfree method for arbitrary evolving cracks. Computer Methods in Applied Mechanics and Engineering, 196(29-30):2777-2799, 2007.

[40] T. Rabczuk and G. Zi. A meshfree method based on the local partition of unity for cohesive cracks. Computational Mechanics, 39(6):743-760, 2007.

[41] T. Rabczuk, P. M. A. Areias, and T. Belytschko. A simplified mesh-free method for shear bands with cohesive surfaces. International Journal for Numerical Methods in Engineering, 69:993-1021, 2007.

[42] K. Ravi-Chandar. Dynamic fracture of nominally brittle materials. International Journal of Fracture, 90(1-2):83-102, 1998.

[43] E. Sharon and J. Fineberg. Microbranching instability and the dynamic fracture of brittle materials. Physical Review B, 54(10):7128-7139, 1996.

[44] J.-H. Song, P. M. A. Areias, and T. Belytschko. A method for dynamic crack and shear band propagation with phantom nodes. International Journal for Numerical Methods in Engineering, 67:868-893, 2006.

[45] J-H Song, P.M.A. Areias, and T. Belytschko. A method for dynamic crack and shear band propagation with phantom nodes. International Journal for Numerical Methods in Engineering, 67(6):868-893, 2006.

[46] M. Stolarska, D. L. Chopp, N. Moës, and T. Belytschko. Modelling crack growth by level sets in the extended finite element method. International Journal for Numerical Methods in Engineering, 51:943-960, 2001.

[47] N. Sukumar, D. L. Chopp, N. Moës, and T. Belytschko. Modelling holes and inclusions by level sets in the extended finite element method. Computer Methods in Applied Mechanics and Engineering, 190(46-47):6183-6200, 2000.

[48] Elguedj T., Gravouil A., and Combescure A. A mixed augmented lagrangian-extended finite element method for modelling elastic-plastic fatigue crack growth with unilateral contact. International Journal for Numerical Methods in Engineering, 71(13):1569-1597, 2007.

Int. J. Numer. Meth. Engng 2000; 00:1-6 
[49] G. Ventura, J. X. Xu, and T. Belytschko. A vector level set method and new discontinuity approximations for crack growth by EFG. International Journal for Numerical Methods in Engineering, 54(6):923-944, 2002.

[50] G. Ventura, E. Budyn, and T. Belytschko. Vector level set for description of propagating cracks in finite elements. International Journal for Numerical Methods in Engineering, 58(10):1571-1592, 2003.

[51] X.-P. Xu and A. Needleman. Numerical simulation of fast crack growth in brittle solids. Journal of the Mechanics and Physics of Solids, 42(9):1397-1434, 1994.

[52] G. Zi and T. Belytschko. New crack-tip elements for XFEM and applications to cohesive cracks. International Journal for Numerical Methods in Engineering, 57(15):2221-2240, 2003.

[53] G. Zi, J.-H. Song, E. Budyn, S.-H. Lee, and T. Belytschko. A method for growing multiple cracks without remeshing and its application to fatigue crack growth. Modelling and Simulation in Materials Science and Engineering, 12(5):901-915, 2004.

[54] G. Zi, H. Chen, J. Xu, and T. Belytschko. The extended finite element method for dynamic fractures. Shock and Vibration, 12(1):9-23, 2005. 